\title{
Direct-drive laser fusion: status and prospects
}

S.E. Bodner, D.G. Colombant, J.H. Gardner, R.H. Lehmberg, S.P. Obenschain, L. Phillips, A.J. Schmitt, J.D. Sethian, R.L. McCrory, W. Seka, C.P. Verdon, J.P. Knauer, B.B. Afeyan, H.T. Powell

\section{January 14, 1998}

U.S. Department of Energy

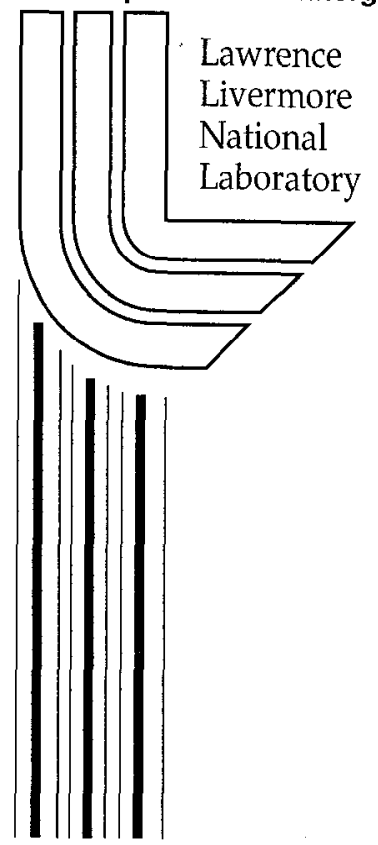




\section{DISCLAIMER}

This document was prepared as an account of work sponsored by an agency of the United States Government. Neither the United States Government nor the University of California nor any of their employees, makes any warranty, express or implied, or assumes any legal liability or responsibility for the accuracy, completeness, or usefulness of any information, apparatus, product, or process disclosed, or represents that its use would not infringe privately owned rights. Reference herein to any specific commercial product, process, or service by trade name, trademark, manufacturer, or otherwise, does not necessarily constitute or imply its endorsement, recommendation, or favoring by the United States Government or the University of California. The views and opinions of authors expressed herein do not necessarily state or reflect those of the United States Government or the University of California, and shall not be used for advertising or product endorsement purposes.

Work performed under the auspices of the U. S. Department of Energy by the University of California Lawrence Livermore National Laboratory under Contract W-7405-Eng-48.

This report has been reproduced directly from the best available copy.

Available to DOF and DOF contractors from the Office of Scientific and Technical Information

P.O. Box 62, Oak Ridge, TN 37831

Prices available from (423) 576-8401

http://apollo.osti.gov/bridge/

Available to the public from the National Technical Information Service

U.S. Department of Commerce 5285 Port Royal Rd., Springfield, VA 22161 http://www.ntis.gov/

OR

Lawrence Livermore National Laboratory Technical Information Department's Digital Library http://www.llnl.gov/tid/Library.html 
PREPRINT

\section{Direct-drive laser fusion: status and prospects}

Stephen E. Bodnert, Denis G. Colombant, John H. Gardner*, Robert H. Lehmberg, Stephen P. Obenschain, Lee Phillips*, Andrew J. Schmitt, John D. Sethian

Plasma Physics Division, Naval Research Laboratory, Washington, DC 20375

* Laboratory for Computational Physics and Fluid Dynamics, Naval Research Laboratory, Washington, DC 20375

Robert L. McCrory, Wolf Seka, Charles P. Verdon, James P. Knauer

Laboratory for Laser Energetics, University of Rochester, Rochester, NY 14623

Bedros B. Afeyan

Polymath Associates, Livermore, CA 94550

Howard T. Powell

Lawrence Livermore National Laboratory, Livermore, CA 94550

Techniques have been developed to improve the uniformity of the laser focal profile, to reduce the ablative Rayleigh-Taylor instability, and to suppress the various laser-plasma instabilities. There are now three directdrive ignition target designs that utilize these techniques. Evaluation of these designs is still ongoing. Some of them may achieve the gains above 100 that are necessary for a fusion reactor. Two laser systems have been proposed that may meet all of the requirements for a fusion reactor.

PACS 52.58Ns

\section{INTRODUCTION}

In the early 1970s, the US laser fusion program was centered upon a simple and elegant pellet concept: a small bare spherical shell of deuteriumtritium fuel (DT) located in the middle of a large empty reactor chamber, directly illuminated by symmetrically positioned laser beams that were carefully tuned to produce high compression and central ignition'. By 1975 this concept had been largely abandoned. The laser beams could not be made sufficiently uniform to compress the shell symmetrically inward; the target design required very high laser intensities with the certainty of triggering numerous laser-plasma instabilities; the implosion was highly unstable to the ablative Rayleigh-Taylor (RT) hydrodynamic instability; and the predicted target gain was too low to overcome the low efficiencies of realistic lasers in a practical reactor.
Although the original target design did not work, the general concept is very appealing: (a) direct illumination of a spherical target maximizes the coupling efficiency from laser energy to imploding DT fuel, and therefore maximizes the potential target gains; (b) the spherical divergence of the plasma corona limits the interaction length between the laser light and the plasma, and thus diminishes the risk of deleterious laser-plasma instabilities; (c) the overall spherical symmetry minimizes the physical complexity of the target and thus limits the number of ways that it can fail; (d) the pellet mass is minimized, which then minimizes the required laser energy, the cost of the pellet, and the mass of the debris.

Through the years there has been a continuing low level of effort on direct-drive laser fusion, and now the above physics problems appear to have been largely resolved. The laser beams can be

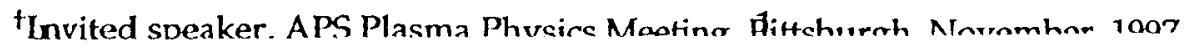




\section{PREPRINT}

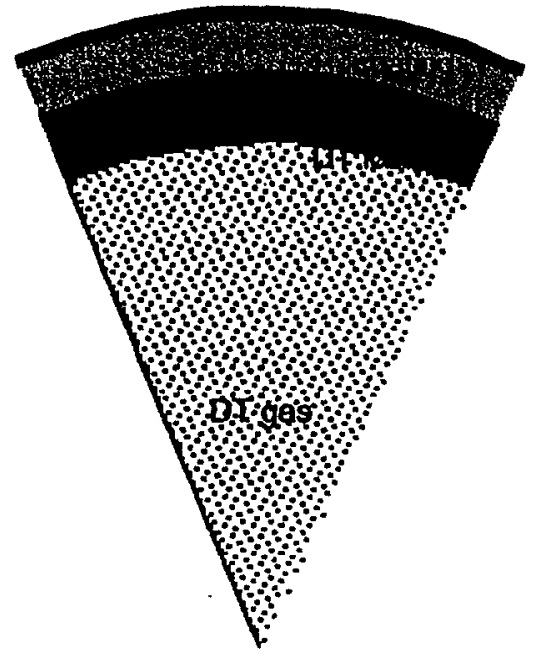

Fig. 1. Direct-drive pellets are geometrically very simple, with approximately equal masses in the DT fuel and the ablator, surrounding a DT gas that is in equilibrium with the fuel. Sometimes there is a thin outer coating.

optically smoothed to produce a residual rms flux nonuniformity that is well below one percent. The risk of laser-plasma instabilities has been minimized by lowering the peak laser intensity, shifting the laser wavelength into the UV, and using optically smoothed laser beams. The RT instability can be ameliorated by preheating the ablator. These techniques have now been pulled together to generate three specific and different direct-drive target designs that may reach ignition. Some may have high enough energy gains to be useful in a fusion reactor. These designs are currently being evaluated and optimized.

This paper will review the status and the prospects of the direct-drive laser fusion concept as of November 1997. We will summarize the experimental and theoretical advances in the field, describe and evaluate the specific target designs, and list some of the problems that remain to be addressed.

Although construction is now underway for a few-shots-per-day megajoule laser for defense applications, there has been very little development of lasers that are suitable for a fusion reactor. These
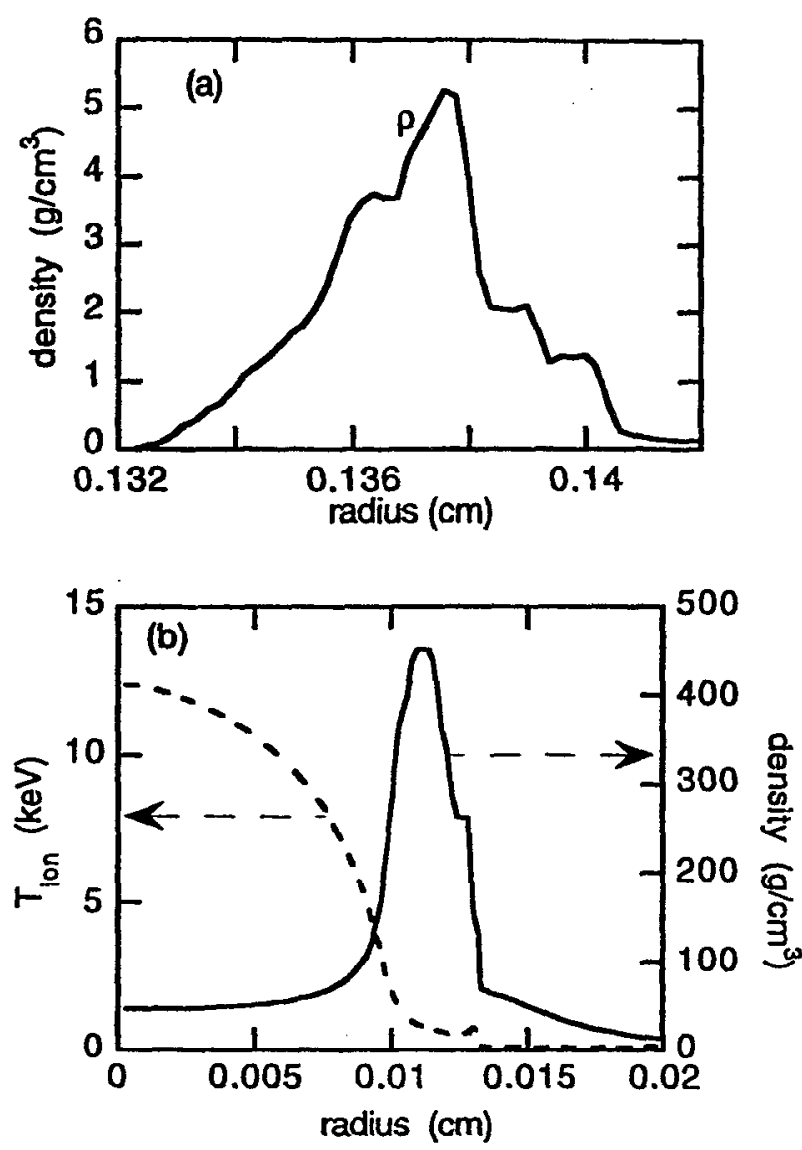

Fig. 2. Typical density profiles for a direct drive pellet, (a) part way in; (b) at final stagnation.

fusion lasers would need a much higher repetition rate, higher durability, higher efficiency, and lower cost. Specific laser concepts have been proposed that may meet all the requirements, using either a krypton-fluoride laser (KrF) or a diode-pumped solid-state laser (DPSSL).

\section{LASER BEAM NONUNIFORMITIES}

\section{A. Laser Beam Uniformity Requirements}

The generic direct-drive pellet is shown in Fig. 1 . A shell of frozen DT fuel (a few hundred microns thick) is surrounded by a low- $Z$ ablator that is burned off by the laser beams. The masses of the DT and the ablator are similar. There is sometimes a thin outer membrane surrounding the ablator. 


\section{PREPRINT}

The pulse shape has two temporal regions: the "foot" and the "main" or "drive" portions. The low-intensity foot of the laser pulse generates a few megabar shock wave that compresses the pellet shell and places it on the desired isentrope. The laser intensity then rises rapidly, generating pressures of 70-100 MBar and accelerating the shell inward. The rising pulse shape is carefully tuned to minimize any additional increases in the isentrope of the DT fuel.

Figure 2a shows a typical density profile during the inward acceleration phase. Figure $2 b$ shows typical density and temperature profiles at final stagnation, with the low-density central spark plug surrounded by a higher-density main fuel region. The hot central region has a $\rho R \sim 0.3 \mathrm{~g} / \mathrm{cm}^{2}$, approximately equal to the range of an alpha particle in a $5 \mathrm{keV}$ spark plug plasma. This spark plug self-heats because of the local alpha particle deposition, rising to a temperature sufficient to initiate a propagating burn into the surrounding colder fuel. The parameters in Fig 2 were taken from recent calculations of a non-optimized directdrive target design at the Naval Research Laboratory (NRL). Note that the spark plug radius $R_{f}$ is $-4 \%$ of the initial pellet radius. If one postulates that any angular variation $\delta R_{f}$ should be no more than $50 \%$ of $R_{f}$, then one can derive ${ }^{2}$ a restriction on the allowable low-mode pressure variations: $\delta P / P \leq 0.5\left(R_{f} / R_{0}\right)$. Since $P \sim 10.8$, one concludes that the very low-mode intensity variations around the pellet should be less than $2.5 \%$.

It has been shown analytically ${ }^{3}$ that almost any even number $(26)$ of carefully located laser beams is sufficient to produce perfect illumination symmetry on a hard sphere. If each beam generates a $\cos ^{2}(\theta)$ intensity distribution on the pellet, then the various $\cos ^{2}$ factors can add to unity. When one includes realistic laser profiles, refraction, collisional absorption, inward motion of the pellet surface, laser beam imbalances, and laser beam mispointing, then the uniformity can no longer be perfect, but more beams will improve the statistics. Studies' have indicated that 60 or more laser beams should meet the low spherical mode $(\ell=1-10)$ symmetry requirements for an implosion.

Higher-mode symmetry criteria are more severe, because the high density shell is being accelerated inward by a lower density plasma corona and the interface is RT unstable. For the simple case of a heavy fluid of density $\rho_{2}$ supported above a lower density fluid of density $\rho_{1}$, and ignoring finite thickness effects, the exponential growth rate of an interface perturbation is given by $\gamma=\sqrt{A k g}$ where $k$ is the mode number of the perturbation, $g$ is the acceleration, and $A$ is the Atwood number $\left(\rho_{2}-\rho_{1}\right) /\left(\rho_{2}+\rho_{1}\right)$. For a spherical implosion, the spherical harmonics are the normal modes, and the analogous formula for large $\ell$ can be approximated by substituting $\ell / R$ for $k$. Thus $\gamma_{0}=\sqrt{A \lg / R}$. If the shell accelerates inwards such that $g t^{2}-R_{0}$, then the number of $e$-folds is proportional to $\sqrt{A l}$. The higher mode numbers, those produced within each laser beam, have the larger growth rate. The modes that are most dangerous will have perturbation wavelengths that are comparable to the pellet shell thickness, since still higher mode numbers would saturate before breaking through the pellet shell. To reduce the mode number of the most dangerous RT modes, target designs try to minimize the in-flight aspect ratio $R / \Delta R$ (average shell radius divided by the inflight shell thickness).

There are several ways of reducing the growth of the most dangerous modes, including density gradient stabilization, ablative (convective) stabilization, nonlinear saturation, mode-mode coupling, and spherical convergence; these will be described later in this article. Figure 3 plots the 


\section{PREPRINT}

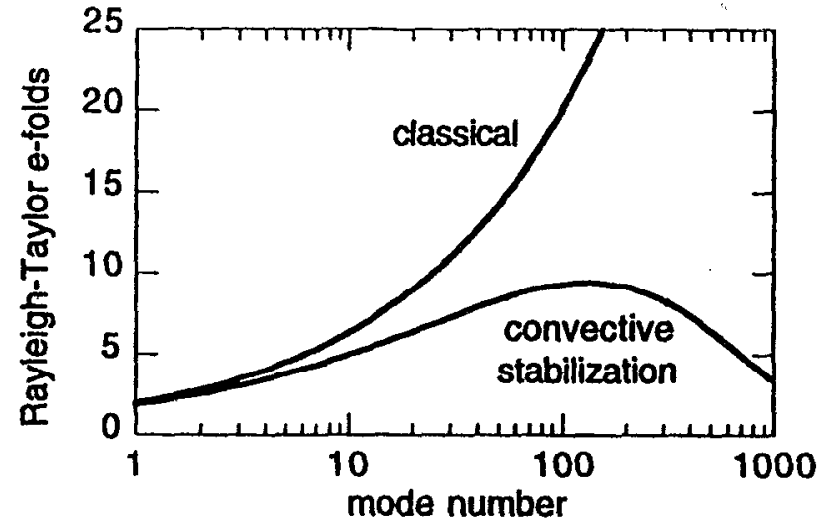

Fig. 3. Typical number of exponentiations of the ablative Rayleigh-Taylor instability during an implosion. The upper "classical" curve assumes a growth rate proportional to $\sqrt{\mathrm{ka}}$. The lower curve includes ablative (convective) stabilization. The maximum growth is near mode number 100 .

classical number of RT e-folds $\int \gamma_{0} d t$, and the value $\int \gamma d t$ with ablative stabilization, versus mode number for a recent target design. The most unstable modes are in the vicinity of 100 . The perturbation wavelength $[\lambda=2 \pi R / \ell=2 R(\pi / \ell)]$ is $\sim \pi / 100$ of the size of an individual laser beam. Since this wavelength is much less than the size of individual laser beams, successful fusion requires methods of reducing the transverse structure within laser beams to a low level:

\section{B. Optical Smoothing of Laser Beams}

\section{Idea of Beam Smoothing (Temporal \& Spatial Bandwidth)}

Large laser systems have many optical elements, each of which introduces phase distortion. At the focal plane, these phase variations would lead to interference effects with large and uncontrollable illumination nonuniformities. The solution to the nonuniformity problem was based on the observation that the most uniform illumination is not coherent laser light, but rather incoherent light from conventional optical sources such as sunlight. Fusion pellets have a diameter of $-5 \mathrm{~mm}$, while the coherent light from a laser can ideally be focused to a spot size of only a few microns. We can therefore trade off the excess coherency and focusability of a laser to achieve better uniformity.

The laser beam is first separated into multiple independently-phased beamlets whose widths $\mathrm{d}$ are small compared to the full beam width $D$. The degree of spatial incoherence is characterized by the large number $N_{S}=D / d$ of beamlets across the beam. For a megajoule class fusion driver, $N_{S}>$ 100. Each beamlet has much less phase distortion than the full beam, and its focal profile can be nearly ideal.

Because of interference between the beamlets in each beam, the instantaneous focal spot intensity produces highly nonuniform random speckle whose envelope is the ideal distribution. The spatial frequencies of this speckle, $n=\left(n_{x}, n_{y}\right)$ form a two-dimensional array of integer multiples. For a square laser aperture, the ensemble-averaged spatial power spectrum is given by

$$
\sigma^{2}(\mathbf{n})=\frac{1}{N_{s}{ }^{2}}\left(1-\frac{\left|n_{x}\right|}{N_{s}}\right)\left(1-\frac{\left|n_{y}\right|}{N_{s}}\right),
$$

where $\left|n_{x}\right|,\left|n_{y}\right| \leq N_{S}$.

Spatial incoherence is then introduced by forcing the speckle patterns to change randomly on a coherence time $\tau_{c}$ that is much shorter than the effective hydrodynamic response time. In the ideal case, where all the beamlet phases change independently, all of the speckle modes are randomized each coherence time, and all are thus smoothed at the same rate. For an effective averaging time $t_{a v}$, there are then $t_{a v} / \tau_{c}$ statistically-independent patterns, and the above equation is replaced by

$$
\sigma^{2}(\mathbf{n})=\left(\frac{\tau_{c}}{t_{a v}}\right) \frac{1}{N_{s}^{2}}\left(1-\frac{\left|n_{x}\right|}{N_{s}}\right)\left(1-\frac{\left|n_{y}\right|}{N_{s}}\right),
$$

thus the speckle nonuniformity approaches zero at sufficiently long averaging times. The total mean 


\section{PREPRINT}

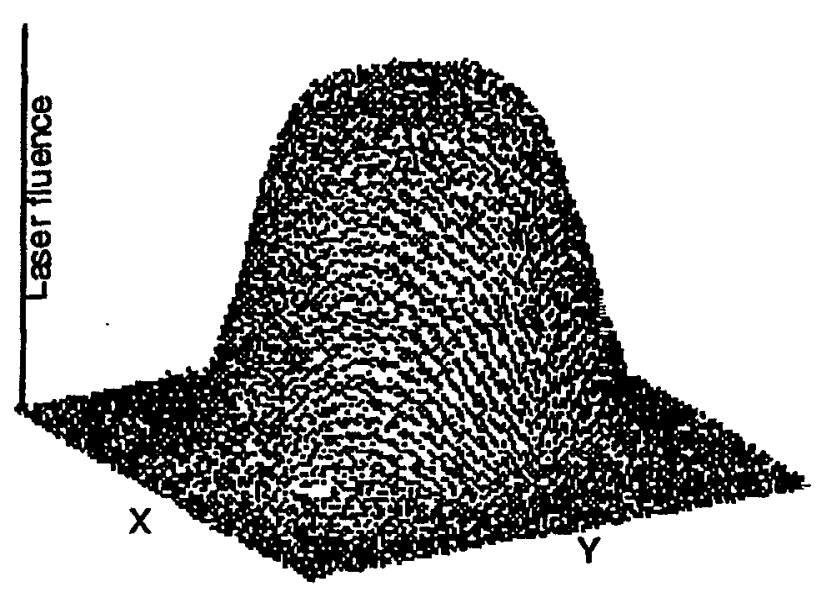

Fig. 4. The measured focal spot laser intensity is very uniform for a $\mathrm{KrF}$ laser beam with ISI smoothing, $1 \mathrm{THz}$ bandwidth, and a $4 \mathrm{~ns}$ averaging time. RMS nonuniformities in each beam are $1 \%$, excluding tilt and curvature.

square nonuniformity becomes $\sigma^{2}=\tau_{c} / t_{a v}$. The effectiveness of the smoothing process thus depends strongly on the laser's bandwidth, with $\Delta v$ $\sim 1 / \tau_{c}$. With the Nike $\mathrm{KrF}$ laser the bandwidth has reached $3 \mathrm{THz}$, with the coherence time as short as 0.35 ps.

\section{Induced Spatial Incoherence}

The most effective beam smoothing scheme that has been tested so far is the Induced Spatial Incoherence (ISI) technique $5,6,7,8,9$. A spatially and temporally incoherent light source uniformly illuminates the front end (object), which is currently chosen to be a hard aperture. (This aperture could also be apodized to create a shaped envelope profile.) The object profile is then imaged through the laser system onto the target to produce the focal spot envelope. To avoid imprinting gain nonuniformities onto the focal spot, each amplifier stage is located at or near the object's (and target's) Fourier transform plane. ISI smoothes all spatial frequencies at the same rate, as described in Eq. 2.

The focal spot envelope will remain relatively insensitive to large scale phase aberration imposed by the laser system if the spot size is many times the diffraction limit, and also large compared to the point-spread distribution (PSD) created by the aberrations. The Nike KrF laser optical design ${ }^{10}$ allows nearly complete compensation of all systematic phase aberrations, such as astigmatism, coma, and spherical aberration. In the present configuration, the PSD width due to random phase aberration is -15 times the diffraction limit. For the 75 XDL focal spots currently being used, this allows a flat top over about half of the envelope, as shown in Fig 4 . The flat region could be extended by using adaptive optics techniques, such as those used in Beamlet ${ }^{11}$ to correct for the phase aberration in the amplifiers.

Using ISI, the Nike KrF laser has produced smooth, flat-topped focal distributions with residual speckle fluctuations of $1 \%$ rms in a single beam with $1 \mathrm{THz}$ bandwidth and $\tau_{\mathrm{c}}=0.66 \mathrm{ps}$ (for a Gaussian spectrum, $\tau_{c} \cong 0.66 / \Delta v$ ). With 37 overlapped Nike beams, the nonuniformity is below that which is measurable, but is estimated to drop by as much as $1 / \sqrt{37}$ to $0.15 \%$ rms (ignoring the short wavelength beam-beam interference). The detailed mode spectrum has been measured for a single beam, and is in agreement with theory ${ }^{12}$. The bandwidth of Nike for some recent target experiments has been extended to $3 \mathrm{THz}$.

Equation 2 can be used to estimate the singlebeam contributions to the low-mode nonuniformities using ISI on a spherical target. For modes $|M| \ll N_{S}$, Eq. (2) reduces to the azimuthallysymmetric expression. The total RMS nonuniformity of all modes up to some value $|M|_{c} \ll N_{S}$ is then

$$
\sigma_{r m s}\left(|\mathbf{M}| \leq|\mathbf{M}|_{c}\right) \cong\left(\frac{\sqrt{\pi}|\mathbf{M}|_{c}}{N_{S}}\right) \sqrt{\frac{\tau_{c}}{t_{a v}}}
$$

If the target diameter equals the focal beam diameter, then the spherical mode number is 
$\ell=\pi|\mathrm{M}|$, and the corresponding RMS nonuniformity of all $\ell$-modes up to $\ell_{c}$ becomes

$$
\sigma_{r m s}\left(\ell \leq \ell_{c}\right) \cong\left[\frac{\ell_{c}}{\sqrt{\pi} N_{S}}\right] \sqrt{\frac{\tau_{c}}{t_{a v}}}
$$

As an example, consider the illumination geometry of the National Ignition Facility (NIF), where a 3.2 mm diameter target (diffraction width $f \lambda / D_{A}=7 \mu m$ ) would require $N_{S}=457$. Without any temporal smoothing, the nonuniformity in all modes $1 \leq \ell \leq 28$ would then be approximately $28 /(457 \sqrt{ } \pi) \cong 3.5 \%$. If, for example, one averages over $100 \mathrm{ps}$ with $\tau_{c}=1 \mathrm{ps}$, then the nonuniformity drops to $0.35 \%$. Thus the speckle from ISI does not introduce significant low-mode nonuniformities.

Because the ISI light is incoherent and chaotic, its speckle varies rapidly in space and time throughout the entire laser system. This is. not a problem in $\mathrm{KrF}$ lasers, where the gaseous medium and low saturation flux ensure very little nonlinear phase distortion in the amplifiers. However it would be undesirable in a glass laser, which can produce large nonlinear phase distortions, and which also requires harmonic conversion.

\section{Smoothing by Spectral Dispersion}

The most promising beam smoothing scheme for solid-state lasers uses instead a technique called Smoothing by Spectral Dispersion (SSD) ${ }^{13}$. Here the spatial incoherence is created near the final focusing lens by a phase plate that breaks the beam into beamlets with adjustable phase shift. The temporal modulation is created near the front end of the laser by a phase modulator with temporal phase dependence $\phi(t)$ followed by a diffraction grating that spectrally disperses the light over an angle $\Delta \theta$. After the grating, the color at any instant cycles across the aperture, and the interference among more distant beamlets averages out in time. Alternatively, one can view the smoothing process as the result of moving the speckle pattern back and forth across the target as the off-axis angle $\boldsymbol{\theta}(t)$ changes in time. The technique has been extended to both transverse dimensions (i.e., 2D-SSD) by adding a second modulator (at a different frequency) and an orthogonal grating to disperse the light in the second transverse direction.

The advantage of SSD is that near the image planes of the gratings the variations are just in the phase, thereby avoiding the self-focusing and nonlinear spectral broadening that could limit high power glass laser amplification. Its main disadvantage so far has been that it cannot effectively smooth the lowest spatial frequency components of the speckle pattern, which arise from interference among nearby beamlets. For $\ell$ values below about 30 , the mode intensities approach the magnitudes given by Eq. (1) rather than Eq. (2).

SSD has now been extended to two transverse dimensions on the OMEGA laser. In addition to the phase plate, there is also a polarization rotator ${ }^{14}$. This rotator is a birefringent wedge placed before the random phase plate to vary the polarization across the beam, reducing the nonuniformities by $1 / \sqrt{2}$. With the polarizer, the single beam nonuniformity is expected to be $8.5 \%$. With the 60 beam of OMEGA, the statistical overlap currently reduces the nonuniformity at the pellet to $-3.5 \%$

Steps are being taken to improve the degree of beam smoothing with SSD. Techniques have been proposed to increase the bandwidth $\Delta v$, which had been limited primarily by dispersion in the sumfrequency output crystal. One can extend the phase matching bandwidth of this crystal by replacing it by a pair of stagger-tuned crystals ${ }^{15}$.

In addition to a broader.bandwidth, the spatial phase variation across the beam (number of color cycles) must be increased to bring the smoothing rate of the longer wavelength speckle closer to the ideal rate described by Eq. 2 . According to recent 
simulations ${ }^{16}$, with increased color cycling the smoothing should approach the ideal rate at nearly all spatial frequencies down to the limit imposed by the dispersion angle $\Delta \theta$.

\section{Other Smoothing Techniques}

The pure ISI technique has also been applied to the Phebus glass laser at Limeil Laboratory ${ }^{17}$. The laser beam uniformity was excellent, and agreed with ISI theory, however the laser had to be operated at reduced energy to avoid nonlinear optical distortion in the amplifiers. At Osaka University ${ }^{18}$ an ISI beam has been propagated through the Gekko XII laser chain, and then passed through a phase plate, producing a very uniform laser beam at the second harmonic.

\section{HYDRODYNAMIC INSTABILITIES}

\section{A. Laser Imprinting and the Rayleigh-Taylor Instability}

The growth of the mass perturbations for each spherical harmonic (ignoring the $m$-variation) can be written as $A_{\ell}=A_{\ell}^{U} \exp \left(\int \gamma_{\ell} d t\right)$. The coefficient $A_{\ell}^{U}$ has four sources from the pellet and laser: (1) perturbations on the inner surface of the DT cryogenic (ice) layer; (2) perturbations on the outer surface finish of the capsule ablator material; (3) long-wavelength laser perturbations associated with beam-to-beam power imbalance, mispointing, and misfocusing; and (4) short-wavelength laser perturbations resulting from imperfections in individual-beam spatial distributions. There is also nonlinear mode coupling.

Success with direct-drive requires that one minimize both the coefficient $A_{\ell}^{0}$ and the growth rate $\gamma_{\ell}$. Since there is often a target performance penalty associated with designing a pellet with lower $\gamma_{\ell}$, the goal has been to first reduce $A_{\ell}^{U}$ to as low a value as possible.
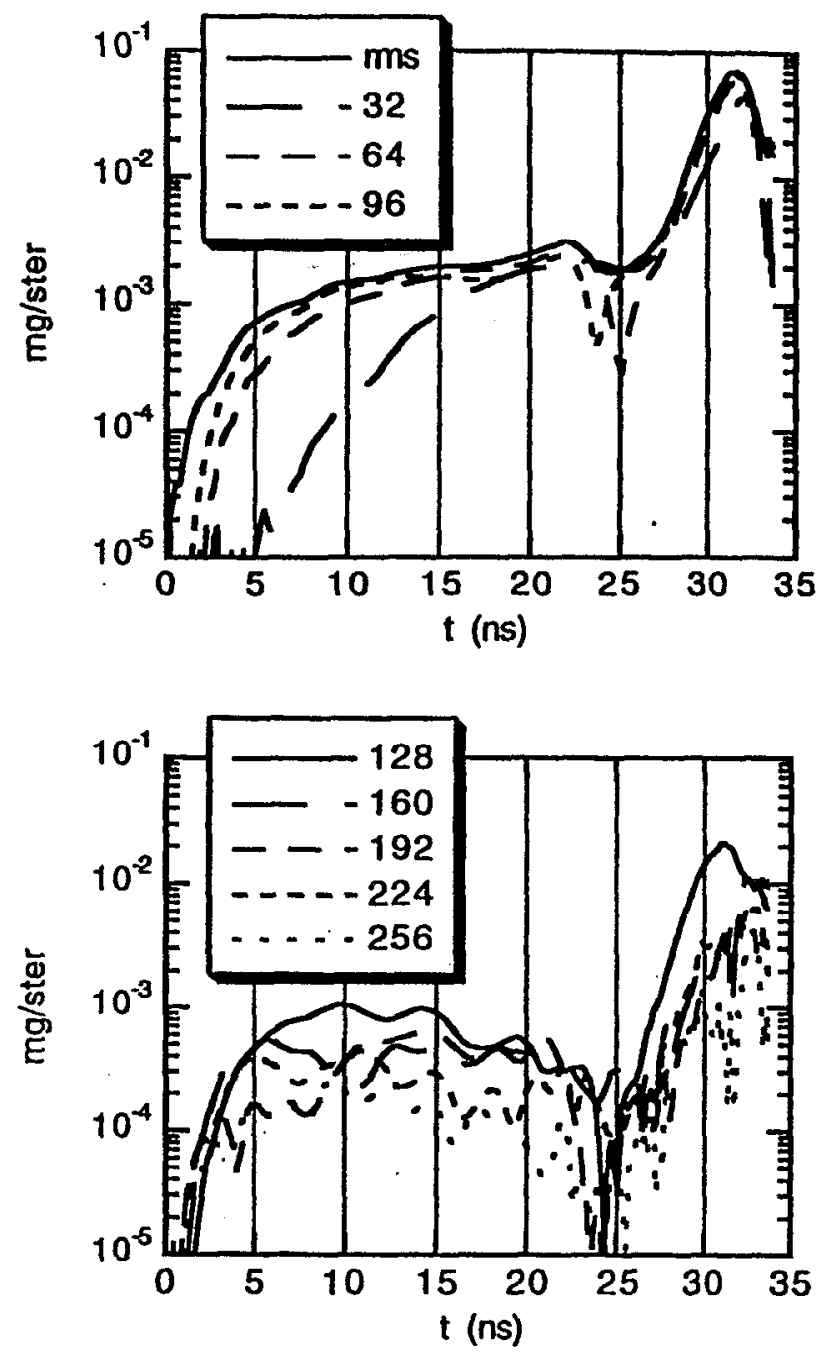

Fig. 5. Typical growth of mass perturbations in various spherical harmonics during a pellet implosion. During the first $20 \mathrm{~ns}$ the laser pulse has a low laser intensity foot, followed by a high intensity main pulse. During the foot the laser "imprints" perturbations. The lower mode numbers, 32, 64 and 96 grow during the entire foot, and rise due to the Rayleigh-Taylor instability. The higher modes, $\geq 128$, are saturated by mass ablation during the foot, and then grow during the main pulse.

The laser fusion program has made considerable progress in the fabrication of smooth pellets. Recent experiments ${ }^{19}$ have shown that it should be possible to limit the roughness on the inner surface of the DT ice to $-1.2 \pm 0.2 \mu \mathrm{m}$. Currently manufactured polymer shells ${ }^{20}$ have an outside surface finish whose roughness is dominated by the lowest 


\section{PREPRINT}

modes. For mode numbers greater than ten, the rms roughness is in the range of $90 \AA$. Some pellet designs now use a low-density foam ablator. The surface finish of foams is currently $\sim 1 \mu \mathrm{m} \mathrm{rms,} \mathrm{but}$ some further improvement is possible ${ }^{21}$. Although full success in target fabrication has not yet been demonstrated in the appropriate geometries, it is possible that target roughness will not be the dominant factor in the coefficient $\gamma_{\ell}$.

If the target fabrication can be sufficiently improved, and if the lasers can be aimed with sufficient accuracy, then the dominant part of $\gamma_{\ell}$ will arise from the inevitable nonuniformities within each of the laser beams. Although the target only responds to a smoother time-average of the laser fluctuations, this time-average is still non zero in any finite time interval. The laser-induced mass nonuniformity" is called "laser imprinting," and is especially large in the earliest portion of the laser pulse, when there is only a thin plasma corona separating the laser absorption surface from the ablation surface. Later in time, thermal conduction and acoustic waves in the thicker corona reduce the laser-imposed nonuniformities.

Figures $5 \mathrm{a}$ and $5 \mathrm{~b}$ show the laser imprinting versus time from a two-dimensional (2D) computer simulation of a pellet implosion that includes a model of the time-dependent ISI laser fluctuations. The higher modes saturate or oscillate early in time, while the lower modes continue to grow during the entire foot of the pulse, which is $\sim 20 \mathrm{~ns}$ in this case.

During the high intensity portion of the laser pulse the shell accelerates inward and the most dangerous perturbation modes grow exponentially due to the RT instability. The largest amplitude perturbations have wavelengths comparable to the in-flight shell thickness, with mode numbers in the range of 50-100. Saturation of these modes occurs either because the spherical convergence closes up the bubbles, or because the target is destroyed.

The early time imprinting on the pellet surface is equivalent to a driven Richtmyer-Meshkov instability, with saturation due to mass ablation ${ }^{23}$. Several methods are under consideration to reduce this laser imprinting. (1) Increasing the laser's bandwidth should reduce the imprint in any given time interval by $\sim 1 / \sqrt{\Delta \omega}$. (2) Lowering the density of the ablator reduces the imprint ${ }^{24}$ by $\sqrt{\rho_{0}}$ (this is somewhat balanced by an increase in the foot duration for the thicker target). (3) Using a longer wavelength laser ${ }^{22}$ during the foot of the pulse increases the separation between the laser absorption region and the dense ablator. (4) Adding a "foam buffer" and a high- $Z$ radiator to the outside of the ablator ${ }^{25}$ creates a smooth supersonic $x$-radiation pulse that produces an early-time plasma corona buffer. (5) Adding a high$Z$ coating directly to the surface of the ablator adds extra free electrons, which also increases the earlytime separation between the absorption and the ablation regions. The application of some of these techniques to specific target designs is described in Section III.

In addition to a minimization of laser imprinting, success with laser fusion requires that the RT growth be reduced to an acceptable level. There are two basic mechanisms to reduce the growth rate: gentle density gradients and convective flow.

If the minimum density gradient scale length $L_{m}=\min |\rho /(d \rho / d x)|$ and the perturbation wave number $k$ satisfy $k L_{m} \gg 1$, then the growth rate is reduced $^{26}$ to $\sqrt{g / L_{m}}$. Radiation can enhance $L_{m}$.

In laser fusion the ablation process convects material through the unstable region. If matter can flow the distance of one perturbation wavelength within one e-folding time then the perturbations are swept out of the unstable region and are convectively stabilized. Thus RT stabilization 


\section{PREPRINT}

should occur if $v_{a} / \gamma_{0}>1 / k$, where $v_{a}$ is mass ablation velocity. One can therefore expect that there would be an approximate RT equation for the growth rate of the form

$$
\gamma=\alpha_{R T} \sqrt{k g}-\beta k v_{a} .
$$

The ablative stabilization concept and the above equation was first derived analytically ${ }^{20}$ from a crude model that predicted, pessimistically, that $\beta=1$. In recent years the community has used a better numerical fit ${ }^{23}, 29,30,31,32$ with $\alpha_{R T}=0.9$ and $\beta=3$. However this newer value of $\beta$ is only applicable when radiation can be neglected ${ }^{32}$. The theory of ablative stabilization of RT has now been extensively studied by several groups $33,34,35,36,37,38,39,40,41,42$ and various detailed formulas have been derived. The newer expressions can still be reasonably approximated by the above equation for $\gamma$, with the coefficients $\alpha_{R T}$ and $\beta$ now dependent upon the target composition. Reference ${ }^{43}$ provides a review of the various models of ablative stabilization, along with useful estimates for the coefficients $\alpha_{R T}$ and $\beta$ in different regimes.

The RT growth can be reduced by increasing the velocity $\mathrm{va}_{\mathrm{a}}$ at the ablation surface. in the second term of the equation. This velocity is related to the density $\rho_{a}$ at the ablation front through the equation for the mass ablation rate $d m / d t=\rho_{a} v_{a}$. To enhance $v_{a}$ one therefore reduces the density $\rho_{a}$ since $d m / d t$ is relatively insensitive to the details of a target design. For a given ablation pressure, the density is reduced when the target is at a higher temperature. Because both the density and temperature of a target vary continuously during the laser pulse, a better parameter to use is the isentrope, proportional to $P / \rho^{\gamma}$. Because the Fermidegenerate pressure varies as $\rho \gamma$, it s convenient to use the isentrope parameter $\alpha=P / P_{F D}$, where $P_{F D}$ is the Fermi-degenerate electron pressure of a fully ionized system. (Note that this isentrope $\alpha$ is not the same as the $\alpha_{R T}$ in the RT equation.)
Designing targets to suppress the RT instability is then equivalent to designing targets with a larger $\alpha$ at the ablation front. However raising $\alpha$ can also reduce the compressibility and the fractional burn up of the DT fuel. For the best fuel compression one wants to keep $\alpha$ near one. Some targets have now been proposed that have a non uniform spatial profile for $\alpha$, maximized in the ablator region and minimized in the DT fuel.

There are two basic methods for adjusting the isentrope of an ablator: radiation preheating and shock preheating. Applications of these techniques to specific target designs are in Section III.

In the first stage of target design we use a 1D spherical radiation/hydrodynamics code, along with post-processed analytic dispersion relations to estimate the RT growth. These RT relations usually include initial imprinting, density gradients, ablative stabilization, and saturation $44,45,66,47,48$. However these RT relations are only approximate, and two-dimensional (2D) or three-dimensional (3D) implosion codes are needed for more accurate analysis. The $2 \mathrm{D}$ computer models we use have a wide variety of physics packages, including the spatial and temporal incoherence of the laser light with specified lens sources, opacities and emissivities of $\mathbf{x}$-radiation from atoms that are not in thermodynamic equilibrium (non-LTE), detailed equations of state for both low and high density materials, nonlinear evolution of $\mathrm{RT}$ into bubbles and spikes, and spherical convergence.

\section{B. Experimental evaluation of laser imprinting and Rayleigh-Taylor}

Because of the inherent limitations in both the numerics and the physics packages of any computer modeling there is of course a need for experiments, especially to test the modeling of laser-imprinting and RT growth. Although directdrive RT experiments have been performed for a 


\section{PREPRINT}

dozen years at various labs, only very recently have experiments been able to combine: (a) improved two-dimensional laser beam smoothing using ISI or 2D-SSD; (b) few-nanosecond laser pulse lengths to simulate the hydrodynamics of a fusion target; (c) improved spatial resolution that can follow the shorter wavelength perturbations. These experiments, summarized below, were performed with the OMEGA glass laser at the University of Rochester Laboratory for Laser Energetics (URLLE), and the Nike KrF laser at the Naval Research Laboratory (NRL).

Since the most dangerous RT wavelengths are comparable to the pellet shell thickness, and small compared to the shell radius, one can reasonably approximate the early-time growth of the perturbations with flat foil targets. To minimize edge effects, these flat foils are only accelerated a distance up to approximately one-half the laser's spot size. Flat foil targets also have the advantage of easier diagnostic access. By creating a separate backlighter that propagates $x$-rays through the foil, one can directly measure the time-dependent transverse mass variations in the target. By using a separate side-on $x$-ray source, one can also directly measure the time-dependent acceleration of the target.

\section{Evaluation with modulated targets}

By first imposing a single-mode sinusoidal perturbation and comparing its growth with code predictions, one can evaluate whether these codes can correctly model the complex hydrodynamic behavior, and whether the laser conditions are as expected.

The recent OMEGA data are shown in Fig. 6. These experiments used a 3 ns square pulse with a peak intensity of $2 \times 10^{14} \mathrm{~W} / \mathrm{cm}^{2}$ on plastic (CH) targets that were $20 \mu \mathrm{m}$ thick, with a $1000 \AA \mathrm{Al}$

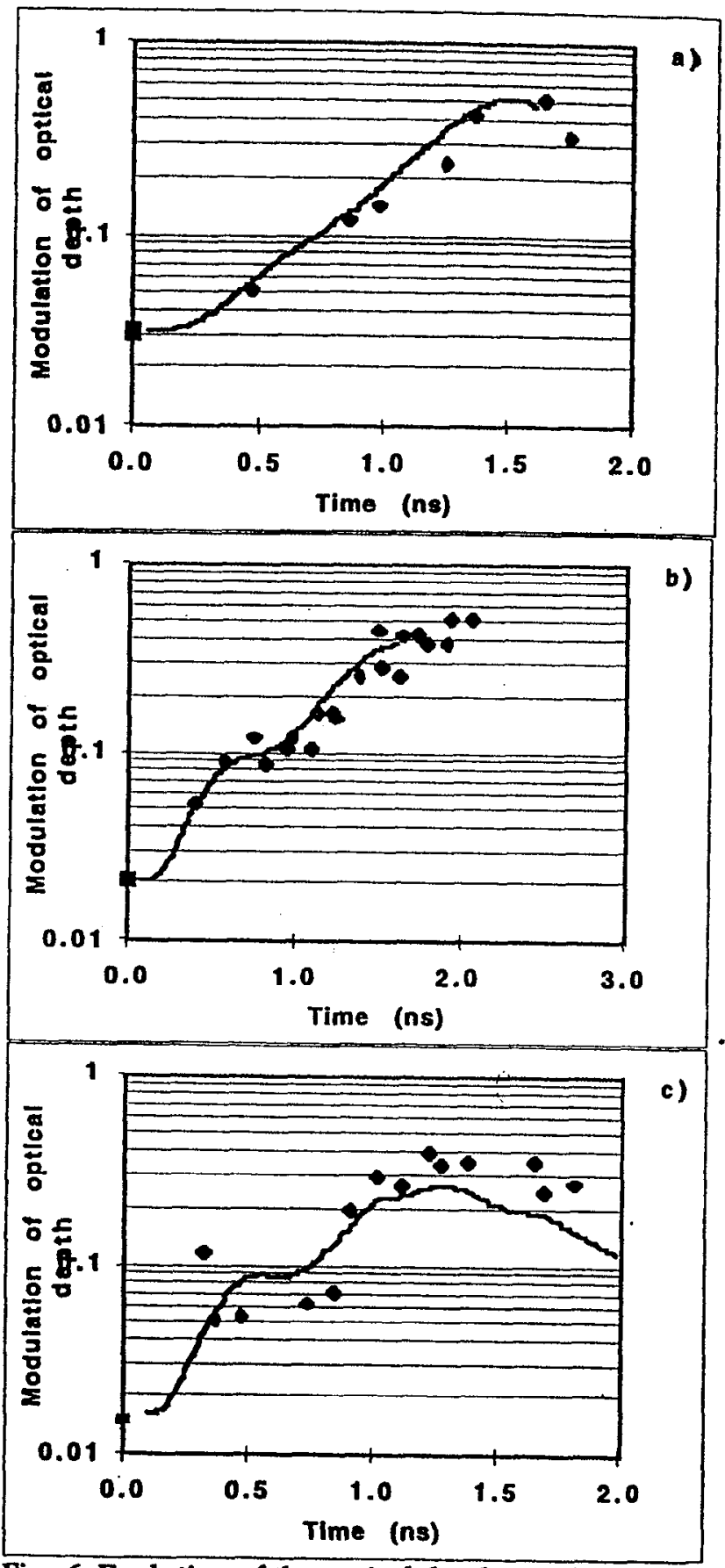

Fig. 6. Evolution of the optical depth as a function of time from an initial perturbation on the ablation surface for planar Rayleigh-Taylor experiments using the OMEGA laser with a 3 ns square laser pulse. Data are shown for (a) $60 \mu \mathrm{m}$, (b) $31 \mu \mathrm{m}$, and (c) $20 \mu \mathrm{m}$ wavelength perturbations. The diamonds are the experimental data and the solid lines are the ORCHID numerical simulations. The square point at time $=0$ is the initial amplitude multiplied by the $x$-ray mass absorption. 


\section{PREPRINT}

overcoat on the laser illumination side to keep the material opaque to the beginning of the laser pulse.

Data are shown for sinusoidal wavelength perturbations of: a) $60 \mu \mathrm{m}$, b) $31 \mu \mathrm{m}$, and c) $20 \mu \mathrm{m}$. The initial amplitudes of these sinusoidal modulations were: a) $0.5 \mu \mathrm{m}$, b) $0.45 \mu \mathrm{m}$, and c) 0.5 $\mu \mathrm{m}$.

The experimental data (plotted as diamonds) and the $\mathrm{ORCHID}^{49}$ predictions (plotted as a line) are in reasonably good agreement for all wavelengths. The rollover of the measured perturbation optical depth at late times has been shown by the simulations to be due to the inability of the diagnostic to resolve the finer spikes of dense material at the ablation surface. The increased scatter in the data for a $20 \mu \mathrm{m}$ wavelength perturbation is due to the resolution limits of the $x$ ray framing camera.

Similar experiments have been performed with the Nike laser using a somewhat lower laser intensity of $8 \times 10^{13} \mathrm{~W} / \mathrm{cm}^{2}$, but with a multinanosecond foot to keep the target on a lower isentrope ${ }^{50}$. The 2D FAST computer simulations ${ }^{51}$ were also in reasonably good agreement with the measured longer wavelength initial perturbations, but deviated from the shorter wavelength perturbations when the long RT spikes are expected to break into a three-dimensional structure that can not be modeled with a 2D code.

The relatively good agreements of OMEGA and Nike experiments with the 2D modeling suggests that these codes can simulate the basic thermal transport and hydrodynamic response of directdrive, finite-amplitude, longer-wavelength initial modulations. There are however three potential weaknesses in the above experiments. The peak laser intensity was only $2 \times 10^{14} \mathrm{~W} / \mathrm{cm}^{2}$, whereas the current fusion targets have a higher peak intensity, $\sim 5-10 \times 10^{14} \mathrm{~W} / \mathrm{cm}^{2}$. At lower intensities the heat transport can be characterized by a
Spitzer-Härm type thermal conductivity ${ }^{22}$. At higher laser intensities and higher electron temperatures the tail of the Maxwellian hot electrons can have a long enough mean free path to directly preheat the target, thereby reducing the RT growth rate. Fokker-Planck computer modeling and RT experiments with short wavelength lasers have not yet fully resolved this uncertainty. Future experiments at URLLE can study the RT behavior at higher intensities by imploding spherical targets.

The second potential weakness in the RT experiments has been the choice of solid $\mathrm{CH}$ as the target material. This was convenient for initial experiments, and provided a useful comparison of our experimental and computational capabilities. Future experiments will shift to the use of the ablator materials to be used in fusion targets: cryogenic DD and DT, DD wicked into CH foams, empty $\mathrm{CH}$ foams, high- $\mathrm{Z}$ coatings, etc.

The third potential weakness is the use of $2 \mathrm{D}$ codes to simulate a $3 \mathrm{D}$ phenomenon. The experimental saturation level of the RT mass modulations is less than the predictions from our 2D codes. This should not provide a basis for optimism. Most likely, the long thin RT spikes break up and flow into the bubble regions, reducing the measured mass nonuniformities. Simulations with NRL's 3 D code ${ }^{53}, 54,55$ predict that the saturation level of the ablative RT instability can be as much as $40 \%$ higher than predicted with 2D codes. The predictions from this 3D code should be taken seriously because they have been successfully compared ${ }^{56}$ with laser imprinting experiments on the VULCAN laser. Until 3D codes are more widely used to design target implosions, a rule of thumb should probably be that 2D target designs should include a margin of safety to account for RT effects.

\section{Evaluation of laser imprinting with smooth targets}




\section{PREPRINT}

The next stage of the experimental program has been to accelerate smooth targets without initial mass modulations, to determine if the 3D laser imprinting of incoherent light from real lasers, and the subsequent RT growth, is adequately modeled by the 2D codes.

With ultra-uniform laser beams, the laser imprinting in the foot of the pulse produces a mass nonuniformity that is too small to directly measure, typically equivalent to $100-1000 \AA$ of surface
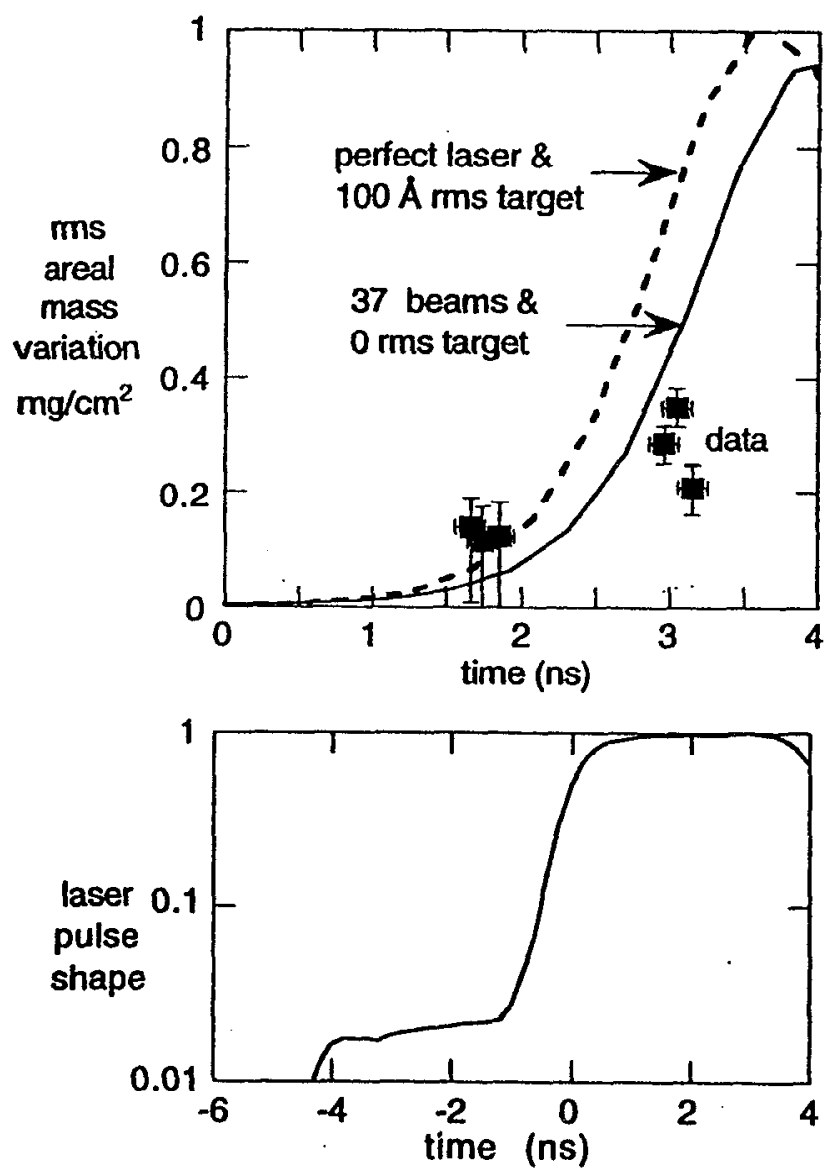

Fig. 7. Measured Rayleigh-Taylor perturbations from acceleration of a smooth planar target. The perturbations arose from imprinting by the Nike laser. The squares are the experimental data and the solid lines are the FAST numerical simulations. The laser pulse was shaped to keep the target on a lower isentrope. The use of a monochromatic backlighter allows the data to be converted from optical depth to $\mathrm{mg} / \mathrm{cm}^{2}$. modulation. The imprinting is only observable after being amplified by the RT instability during the main portion of the laser pulse. In these experiments the RT instability is therefore used primarily as a diagnostic of the imprinting.

Figure 7 shows some measurements of the RTamplified imprinting using 37 overlapped Nike laser beams. These $\mathrm{CH}$ targets were $40 \mu \mathrm{m}$ thick, with a very small initial surface roughness (20-30 $\AA$ rms). A 3-4 ns foot on the Nike pulse with amplitude of $2 \%$ of the main pulse simulated the long low intensity foot of high gain target designs. This foot was followed by a 4 ns main pulse that accelerated the target at $0.8 \times 10^{14} \mathrm{~W} / \mathrm{cm}^{2}$. The foot and main pulses were chosen such that the shocks arrive almost simultaneously at the rear side of the target, thereby keeping the target on a low isentrope ( $\alpha$ is predicted to slowly increased from 2 to 4 during the main pulse).The experiment employed a spherically-curved crystal imaging optic ${ }^{57}$ with the $x$-ray backlighter. This technique produces a nearly monochromatic image of the backlit target (at $1.86 \mathrm{keV}$ ) so that the optical depth modulations could be converted into absolute areal mass nonuniformities.

Figure 7 shows the areal mass nonuniformities from three laser shots compared with two FAST 2D simulations: (a) ISI laser imprinting with a perfect target; and (b) perfect laser beams with a target that had a $100 \AA \mathrm{rms}$ initial surface roughness (laser illumination side). There is relatively good agreement between experiment and the ISI simulation modeling for the time of appearance of measurable mass nonuniformities (more evident if Fig. 7 were replotted on a log scale). The saturation amplitude is however 2-3 times smaller than predicted. This difference may be due to $3 \mathrm{D}$ effects at the shorter transverse wavelengths that can not be modeled by a $2 \mathrm{D}$ simulation. The results also indicate that the laser imprinting can be very small 


\section{PREPRINT}
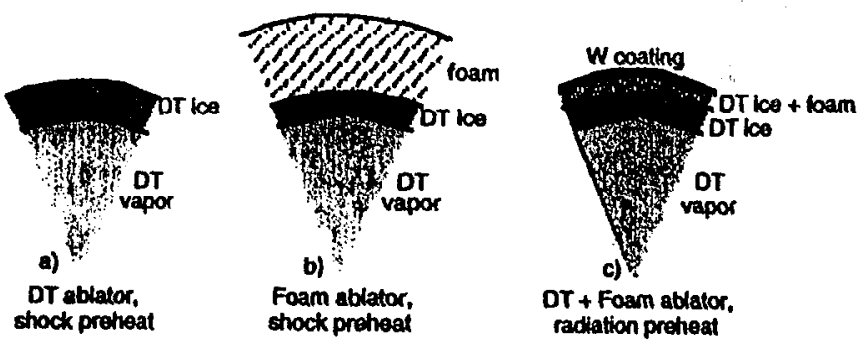

Fig. 8. Three possible direct drive target designs that may reach ignition. (a) An all DT design with shock preheating; (b) A low density $\mathrm{CH}$ foam ablator with shock preheating; (c) An ablator of $\mathrm{CH}$ foam plus frozen DT, preheated by radiation from a tungsten coating.

with state-of-the-art beam smoothing, equivalent to about $100 \AA$ rms initial surface roughness for these Nike parameters.

\section{IGNITION AND HIGH-GAIN TARGET CONCEPTS}

Figure 8 illustrates three different pellet concepts for direct-drive laser fusion. The first design using a DT ablator was proposed by $\mathrm{C}$. Verdon of URLLE at the November 1993 APS Plasma Physics meeting ${ }^{58}$ and is published here for the first time. The intensity of the first shock has been raised to put the DT on a higher isentrope. Typically, the DT has an isentrope $\alpha \sim 2-3$. This target is calculated to have acceptably low laser imprinting and RT growth, with an energy gain of 20 using a $1.5 \mathrm{MJ}$ laser such as the NIF. This target has two major advantages. First, because the ablator and the fuel consist of the same material, there is no risk of a classical RT instability at the ablator/fuel interface. Second, making the ablator out of DT maximizes the rocket efficiency of the implosion, and thus maximizes the potential energy gains. There is however one basic disadvantage. Because the shock preheating of the ablator also raises the isentrope of the fuel, there is reduced fuel compression and thus reduced energy gains, compared to keeping the DT fuel on a lower isentrope.
In the second target the ablator has been replaced with a low density $\mathrm{CH}$ foam. The foam is again preheated with an initial shock, in a fashion similar to the previous target. However the ablator can now be put on a higher isentrope than the DT fuel. With this spatial profile modification of $\alpha$, one can hopefully enhance RT control while at the same time minimizing the preheat of the fuel. This target has been proposed and is being evaluated by $A$. Schmitt and J. Gardner of NRL. It is described in greater detail in Ref. 51. Implosion studies with FAST2D indicate that the combined laser imprinting and RT growth are acceptable, with target gains in the range of 50 for a $1.5 \mathrm{MJ}$ laser and 80 for a $3.5 \mathrm{MJ}$ laser. The potential disadvantage of this design has been its sensitivity to the outer surface finish of the low-density foam ablator.

The ablator in the third target is DT wicked into a low-density $\mathrm{CH}$ foam, along with a coating of a high- $Z$ material such as tungsten. Instead of shock preheating, it uses radiation preheating. During the low intensity of the foot pulse, tungsten emits strongly at 200-250 eV, below the K-edge of carbon. This radiation penetrates deeply into the ablator. This target has the advantage that the fuel can be kept on a very low isentrope while the ablator is on a high isentrope. The tungsten blows off during the foot of the laser pulse, and the main laser pulse directly illuminates the mostly-DT ablator, thus maintaining the advantages of the first target. Because the mass of the ablator is mostly DT, it is less sensitive to the surface finish of the foam. This third design is proposed by S. Bodner and J. Gardner of NRL and is now being more thoroughly evaluated. The potential disadvantages are not yet known.

\section{A. Target design with shock-preheated DT ablator}




\section{PREPRINT}

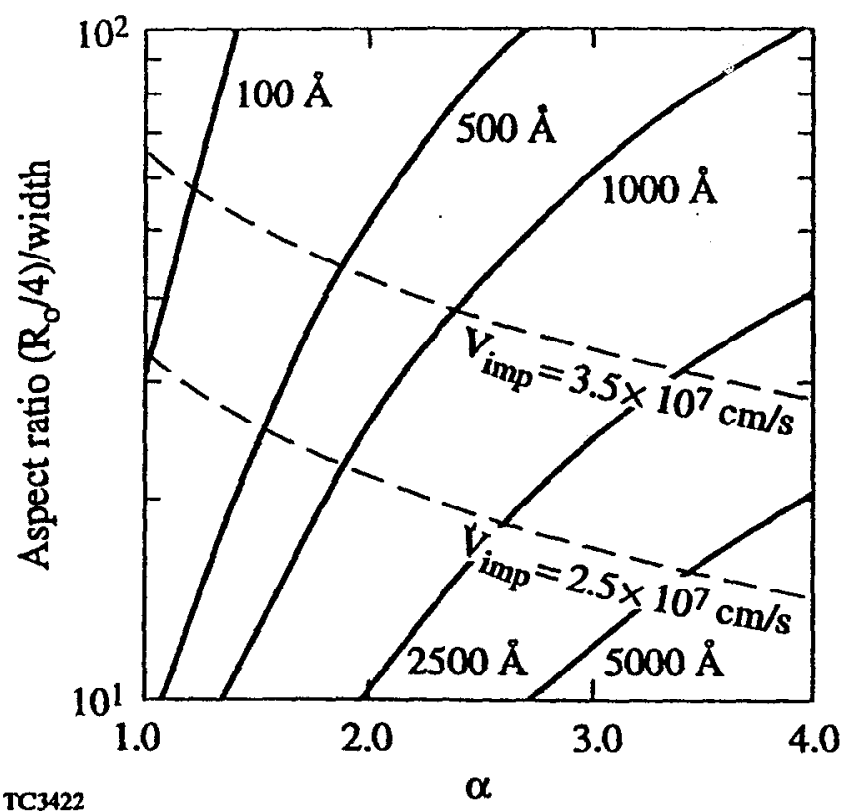

Fig. 9. Solid curves: inverse mix-region width of a pellet [expressed in terms of an in-flight ratio $\left(R_{0} / 4\right) / \Delta r_{\text {mix }}$ ] as a function of degeneracy parameter $\alpha$ for a number of initial perturbation amplitudes. Dashed curves: in-flight aspect ratio $\left(R_{0} / 4\right) / \Delta r_{\text {shell }}$ as a function of $a$ for capsule designs with two different implosion velocities $V_{\text {imp. }}$ For a given $a$, the initial perturbation amplitude should be small enough that the operating point on the solid curve lies above the dashed curve (i.e., $\Delta r_{\text {mix }}<\Delta r_{\text {shell }}$ ).

\section{Simplified Model to Identify Acceptable Design Space}

A very simplified constant-acceleration model can be used to quickly estimate, for a given RT instability model, the amount of perturbation growth a direct-drive capsule design will experience during the acceleration phase and how this growth compares to the over-dense shell thickness during the implosion.

As discussed above, the parameters $\alpha_{R T}$ and $\beta$ in the dispersion relation for the $\mathrm{RT}$ growth rate are dependent on the material composition of the ablator. For a nominal density $\mathrm{CH}$ ablator $(\rho=$ 1.07), detailed simulations have show that typically $\alpha_{R T}=0.98$ and $\beta v_{a}=2.6 \times 10^{5} \mathrm{~cm} / \mathrm{s}$. For a DT ablator $(\rho=0.25), \alpha_{R T}=0.95$ and $\beta v_{\mathfrak{a}}=8.1 \times 10^{5}$ $\mathrm{cm} / \mathrm{s}$. The higher value for $\beta v_{a}$ is due to a higher ablation velocity and a steeper scale length. For direct-drive implosions of solid density material, DT is the optimum ablator for suppression of the RT ablation surface instability. In addition, simple scaling arguments and detailed numerical simulations have found that for directly driven implosions the ablation velocity scales as $v_{a} \propto \alpha^{3 / 5}$, where $\alpha$ is the isentrope. Using this information, along with the saturation model of Ref. 44, a simple model can be developed for the tolerable level of initial nonuniformity.

This model assumes a constant acceleration from the initial radius $R_{0}$ to $R_{0} / 4$, (at which point the imploding shell begins to thicken due to spherical convergence), and a spectrum of the initial nonuniformity, assumed for this example to be of the form $\zeta(\ell)=\zeta_{0} \ell^{-1}$ where $\zeta(\ell)$ is the perturbation amplitude for the Legendre mode $\ell$. The model calculates the width of the Rayleigh-Taylor mix region $\left(\Delta r_{m i x}\right)$ as a function of time.

Results are shown in Fig. 9: The solid curves give $\left(R_{0} / 4\right) / \Delta r_{\text {mix }}$ as a function of the isentrope $\alpha$ for various initial perturbation amplitudes $\zeta_{\mathrm{o}}$ from 100 $\AA$ to $5000 \AA$. For a given $\zeta_{0} \Delta r_{\text {mix }}$ decreases as the departure from Fermi degeneracy $(\alpha)$ increases, since the increased ablation velocity leads to reduced $\mathrm{RT}$ growth. The dashed curves give $\left(R_{0} / 4\right) / \Delta r_{\text {shell }}$ for two capsule implosion velocities $v_{i m p}$ close to $3 \times 10^{7} \mathrm{~cm} / \mathrm{s}$. For a given $v_{i m p}$, the intersections of the solid curves with the dashed curve give the maximum permissible $\zeta_{0}$ as a function of $\alpha$.

As an illustration, a capsule design with a isentrope $\alpha=1$ in the fuel and ablator, and an incident energy of $1.5 \mathrm{MJ}$, produces an implosion velocity between 2.5 and $3.5 \times 10^{7} \mathrm{~cm} / \mathrm{s}$. Figure 9 shows that the maximum perturbation amplitude must be less than $\sim 100 \AA$ if the mix thickness $\left(\Delta r_{\text {mix }}\right)$ is not to exceed the over dense shell thickness $\left(\Delta r_{\text {shell }}\right)$ during the implosion. This 
amplitude represents the combination of capsule surface finish and initial laser imprinting added in quadrature. As the isentrope $\alpha$ is increased, the amount of perturbation that the capsule can withstand increases. For the incident energy range of approximately $1.5 \mathrm{MJ}$, ignition and moderate gain require an $\alpha$ no greater than 3 to 4. From Fig. 9 the maximum initial perturbation amplitude must then be less than $5000 \AA$.

Detailed two-dimensional stability calculations have shown that the results from this simple model are reasonably accurate for the acceleration phase of the implosion. When the earlier portions of the capsule implosion are accurately treated, the initial perturbations must be reduced by a factor of about 2 to 3 from those shown in Fig. 9, because there is a small additional amount of continual growth of longer-wavelength perturbations during the foot of the pulse. When the Rayleigh-Taylor growth of initial inside-surface perturbations during the deceleration phase is also included, the acceptable initial perturbation must be reduced by another factor of approximately 2 . Thus, the initial perturbation amplitude must be $<1000 \AA$ when $\alpha=$ 3.

While obviously not a replacement for actual multidimensional simulations, this model has been found to be an accurate indicator of whether a single-shell direct-drive capsule is likely to experience excessive shell distortions during the implosion phase and/or what the level of "initial" perturbations must be limited to in order to avoid shell disruption during the implosion.

\section{Ignition and High-Gain Single-Shell Designs}

In this section we will conșider a series of "allDT," direct-drive, single-shell cryogenic capsules designed for the incident energy range expected for the NIF; 1.5 to $1.8 \mathrm{MJ}$ at $351 \mathrm{~nm}$. These NIF designs incorporate very thick layers of DT, which act as both fuel and ablator and are surrounded by a very thin layer of normal-density polymer material. (The polymer is required to contain the DT vapor pressure. It is unimportant for the target physics.)

A number of one-dimensional, direct-drive, point-design calculations have been carried out. Figure 10 displays the results for four values of $\alpha$. (The value of $\alpha$ is the mass-weighted average over the cold main fuel layer at a time close to ignition.) In Fig. 10 tangential focusing is assumed: (i.e., the foci of the individual laser beams are fixed such that the envelope of each beam covers an entire hemisphere of the initial capsule surface).

On the basis of the simplified model presented above, a conservative approach to designing an ignition-and-gain direct-drive capsule design for the NIF would begin with an $\alpha$ between 3 and 4 . The inner and outer radii of the $0.25 \mathrm{~g} / \mathrm{cm}^{3}$ DT material are $1350 \mu \mathrm{m}$ and $1695 \mu \mathrm{m}$. For this "all$\mathrm{DT}^{\prime \prime}$ design there is an outer polymer $(\mathrm{CH})$ layer with thickness of $4 \mu \mathrm{m}$. The initial section of the temporal pulse shape-the foot-has been

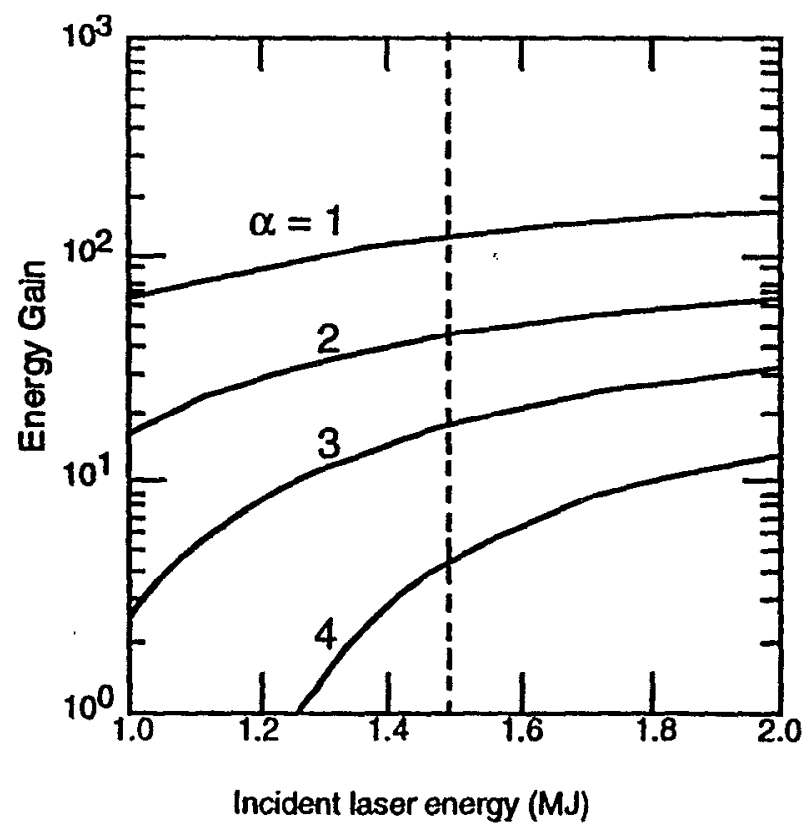

Fig. 10. Pellet gain versus laser energy for the all$D T$ target, for four values of the isentrope parameter $\alpha=P / P F D$ 


\section{PREPRINT}

designed to both place the capsule on the desired implosion isentrope and minimize the development of the Rayleigh-Taylor instability during this foot portion of the implosion. The power during the foot rises very rapidly from "zero" to a constant power of 10 TW and is held constant for $5 \mathrm{~ns}$. The main drive portion is temporally shaped to achieve the desired implosion velocity while minimizing further preheating of the main fuel layer. The drive portion has a temporal duration of approximately $5 \mathrm{~ns}$ with the major portion of the energy delivered in a $2.55 \mathrm{~ns}$ section with a peak power of 475 TW. The one-dimensional gain calculated for this design is $\sim 20$ - twice that quoted for indirect-drive capsule designs at the same incident energy.

The results presented above are from onedimensional simulations. The next step is to address the growth of the perturbations. A large number of two-dimensional numerical simulations have been carried out, using one perturbation wavelength at a time for a selection of modes $\ell$ in

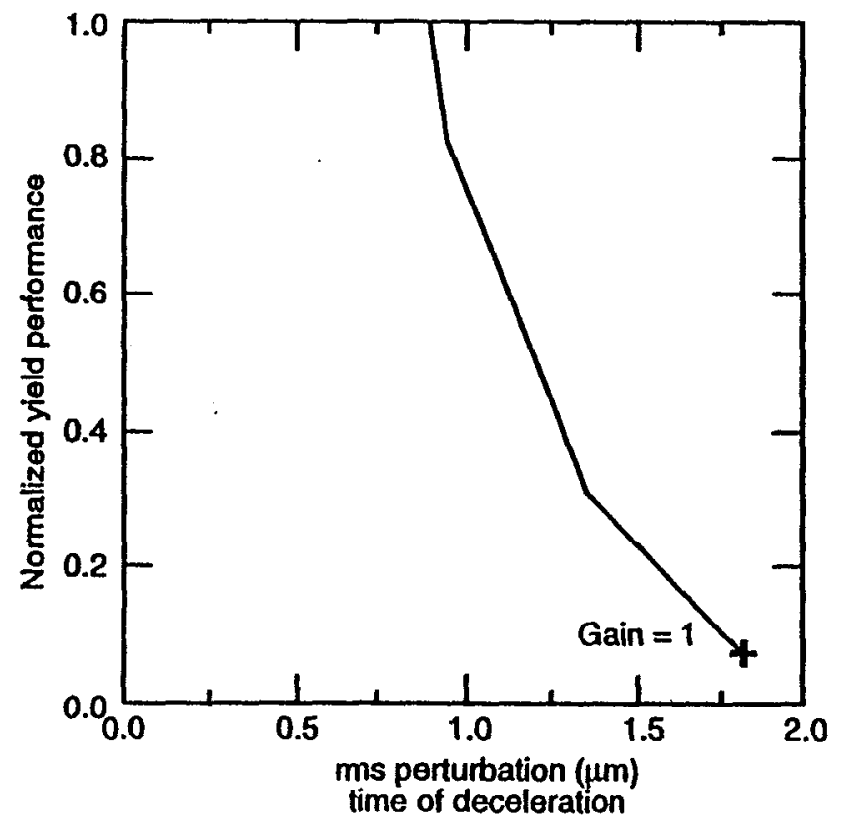

Fig. 11. For the all-DT target, yield versus rms perturbation at the inner surface of the DT at beginning of pellet deceleration, showing that this perturbation should be less than $1.5 \mu \mathrm{m}$ for ignition. the range of 10 to 1000 . The time-dependent irradiation nonuniformity in each mode was obtained from a (simplified) two-dimensional model for 2-D SSD that agrees with more-detailed three-dimensional modeling of the time-dependent smoothing. Typically 50 to 80 individual perturbation wavelengths were modeled for each capsule design.

The evolution of these modes was then combined with a saturation model to determine the temporal evolution of the unstable mix thickness during the capsule implosion. The predicted rear-surface mode spectrum was then used in full twodimensional simulations to determine at what point the capsule fails to ignite and burn (assuming the shell did not totally break up during the acceleration phase). Figure 11 displays the results of these calculations for the $\alpha=3$ design. The simulations showed that if the amplitude of the inner surface perturbations during the deceleration phase exceeded $1.5 \mu \mathrm{m}$ rms, the capsule would fail to ignite. Using these results, one can arrive at a "trade-off" with respect to the levels of initial perturbations the $\alpha=3$ design can tolerate and still ignite and burn. Using the $1.5 \mu \mathrm{m}$ inner surface requirement in conjunction with modal spectrums measured for the outside-surface initial-surface finish, and the two-dimensional imprinting simulations, one finds that the combined perturbations associated with initial-surface nonuniformities and imprinting must be maintained below $1000 \AA$ for this capsule to still ignite and burn (a value very close to that obtained using the simplified model). A "trade-off" can be performed if the sources of perturbations are independent and therefore add in quadrature. If one assumes $100 \AA$ and a $750 \AA$ initial polymer surface finishes then the level of imprinting that will still ignite is respectively $995 \AA$ and $660 \AA$. Direct two-dimensional numerical simulations of 


\section{PREPRINT}

the imprinting with 2-D SSD on this capsule design find a lower imprint level of $\sim 400 \AA$ to $500 \AA \mathrm{rms}$. Thus this capsule design does have the potential to ignite and burn on the NIF. The two-dimensional simulations have also found that at these levels of imprinting the capsule will provide essentially 1D yield performance.

This target design has also been evaluated independently with the NRL 1D and 2D implosion codes. Some differences have been found in the timing and behavior of shocks that bounce off the origin, but the target pulse shape can be readjusted to produce ignition with similar laser nonuniformities.

Some improvements in both target gain and stability have been investigated at URLLE. One design under consideration replaces the portion of the DT capsule that is ablated by the laser with a DT-wetted polymer foam matrix. This results in an increase in gain to approximately 50 (due to higher laser-energy absorption) with little or no degradation in stability. Additional design modifications aimed at reducing the levels of imprinting are being actively pursued.

\section{B. Target design with shock-preheated foam ablator}

\section{Pellet Design Parameters}

A different approach to controlling the RT instability uses an ablator consisting of low-density $\mathrm{CH}$ foam. For a given pressure (e.g. the first shock) the density is now lower and the isentrope is higher compared to a solid density $\mathrm{CH}$ ablator. Shocks moving from the ablator into the DT will partially reflect off the interface due to the lower shock speed in the DT. This reflected shock then heats and compresses the ablator a second time.
For example, a $50 \mathrm{mg} / \mathrm{cm}^{3}$ foam would be shocked a factor of 12 times to $0.6 \mathrm{~g} / \mathrm{cm}^{3}$ after the first shock, while the $0.25 \mathrm{~g} / \mathrm{cm}^{3}$ DT fuel would increase to $\sim 1 \mathrm{~g} / \mathrm{cm}^{3}$.

A possible problem with this approach is that the fuel/ablator interface is classically RT unstable. However if this density ratio is small enough after the initial compression, then the RT growth may be acceptable. For the above situation, the Atwood number is $\left(\rho_{2}-\rho_{1}\right) /\left(\rho_{2}+\rho_{1}\right)=0.4 / 1.6=0.25$. With this low Atwood number the 2D simulations show that the interface RT instability is not important.

Figure $8 \mathrm{~b}$ illustrates this target design. There is a thin vapor barrier between the two materials that has been ignored for simplicity in the simulations. The studies have emphasized a laser energy of $\sim 3.5 \mathrm{MJ}$, to examine the potential of higher energy gains. In one example, the inner radius of the 0.25 $\mathrm{g} / \mathrm{cm}^{3} \mathrm{DT}$ is $1800 \mu \mathrm{m}$. The inner and outer radii of the $\mathrm{CH}$ foam are $2077 \mu \mathrm{m}$ and $2947 \mu \mathrm{m}$. The foot of the pulse rises quickly from zero to $3 \mathrm{TW}$ (producing 2.75 $\times 10^{12} \mathrm{~W} / \mathrm{cm}^{2}$ ), shocking the DT to about $2 \mathrm{MBar}$. The foot of the pulse then rises slowly for $20 \mathrm{~ns}$, reaching half power at $26.4 \mathrm{~ns}$ and full power of $440 \mathrm{TW}$ at $28.1 \mathrm{~ns}$, then remaining constant for $7 \mathrm{~ns}$.

The mass-averaged shell velocity reaches $3.3 \times 10^{7} \mathrm{~cm} / \mathrm{sec}$ before stagnation and compression to $460 \mathrm{~g} / \mathrm{cm}^{3}$. The $1 D$ gain is approximately 80 . (Recent 1D simulations with a lower energy 1.5 MJ laser predict gains of 50.) Higher gains should be possible with optimized pulse shaping and with "zooming" of the laser beams. (With ISI, one can easily reduce the laser focal spot size during the implosion to better match the size of the imploding pellet $^{6}$.) Sample density profiles for this target were shown in Figs. 2a and $2 b$. 


\section{PREPRINT}

\section{2D Stability Analysis}

This target design was analyzed with NRL's FAST2D Eulerian sliding-zone implosion code ${ }^{51}$. This code can simultaneously evaluate several perturbation modes and follow them into the nonlinear regime. Figure 12 shows one of the 2D calculations of laser imprinting and RT growth using a $2 \mathrm{THz} \mathrm{KrF}$ laser with ISI. The bubbles do not break through and eventually are squeezed out due to the spherical convergence. The inner surface of the shell maintains its integrity. Separate calculations show that the target also maintains its integrity if the DT inner surface roughness is less than $0.5 \mu \mathrm{m} \mathrm{rms}$. The most sensitive part of this design is apparently the requirement on the outer surface finish of the foam. The target imploded successfully in the calculations when the outer surface perturbation was $500 \AA \mathrm{rms}$, but failed when the surface perturbation was $5000 \AA$ rms. The best foams consist of thin fibers that are separated by about $1000 \AA$. Thus target fabrication may be the limiting constraint on the success of this type of

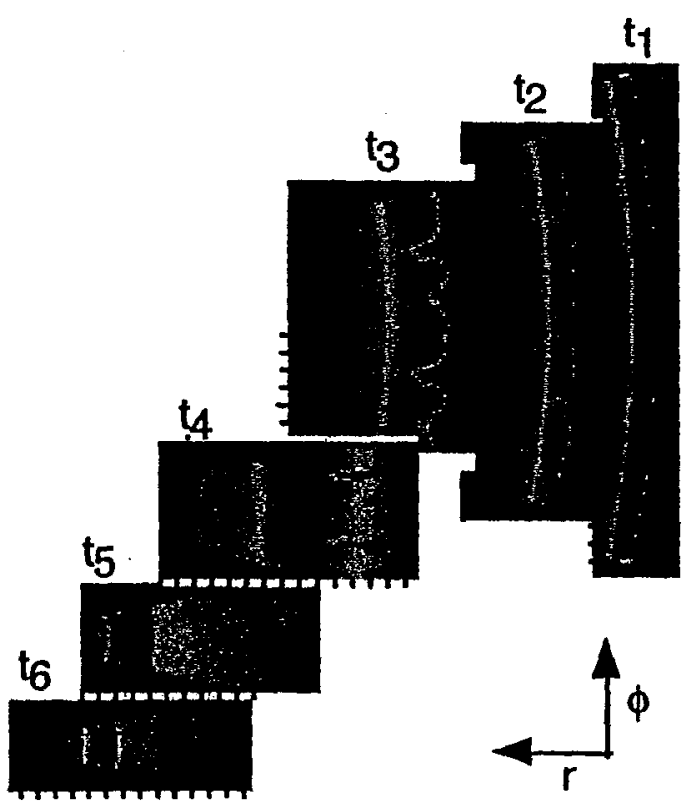

Fig. 12. Density structure at six different times during the implosion of the target with a $\mathrm{CH}$ foam ablator. target.

Figure 13 provides a useful plot of the minimum and angle-averaged shell masses per steradian, where the mass modulations arise from laser imprinting (time moves from right to left as the shell implodes and the radius decreases). The shell mass in the bubble region is only $20 \%$ less than the angle-averaged shell mass. Later in time the bubbles close up and the shell mass in the bubble region approaches the angle-averaged mass.

This implosion was recalculated with a bandwidth of $0.3 \mathrm{THz}$, with all other parameters the same. The inner surface still maintained its integrity, although the shell mass in the bubble region dropped to $40 \%$ of the average shell mass. The target still successfully implodes, although one would expect degraded performance.

\section{Target design with radiation-preheated DT/CH-foam ablator}

The third target concept uses tungsten to preheat the ablator with soft $x$-rays. This ablator can not be pure DT, because the opacity of DT is too low to efficiently stop $x$-rays. The ablator can not be solid $\mathrm{CH}$, because the opacity is then too high, and the

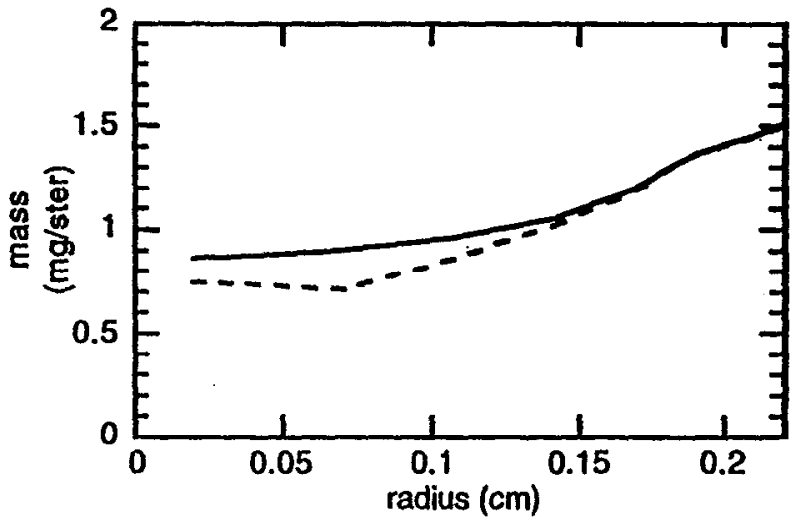

Fig. 13. For the target with a $\mathrm{CH}$ foam ablator, average (solid line) and minimum (dashed line) mass per steradian during the implosion. Time proceeds from right to left as the pellet radius decreases. 


\section{PREPRINT}

soft $\mathbf{x}$-rays would only penetrate into the outer part of the ablator. We therefore propose to use a mixture of low-density $\mathrm{CH}$ foam wicked with frozen DT. The ratio of $\mathrm{CH}$ foam mass to DT mass can be adjusted to optimize the opacity of the ablator. For sub kilovolt $x$-rays the minimum opacity of carbon occurs just below its K-edge: 284 $\mathrm{eV}$ for cold carbon, (rising to $\sim 300 \mathrm{eV}$ at higher electron temperatures). When tungsten is heated to $50-70 \mathrm{eV}$ by the foot of the laser pulse, it is an excellent emitter of radiation near $250 \mathrm{eV}$, with $\sim 50$ $\mathrm{eV}$ width. The tungsten radiation lasts only a few nanoseconds, after which it is blown off.

The detailed design of this target had to await the development of improved non-LTE opacity models that can evaluate the tungsten. There have been several recent improvements in non-LTE opacity modeling", along with practical techniques for rapidly calculating opacities in a radiationhydrodynamics code, and calculations of this target have begun. The optimum thickness of the

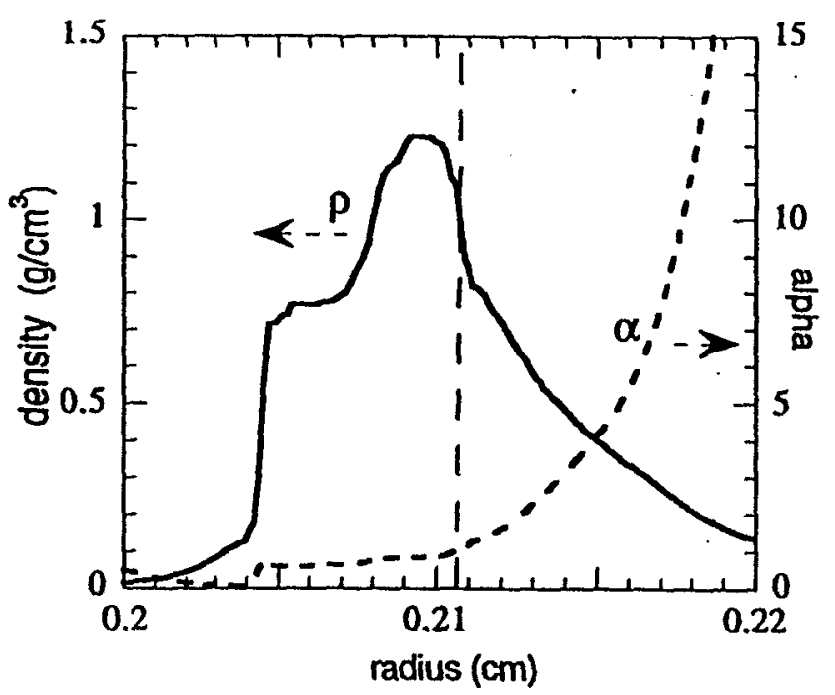

Fig. 14. Density $\rho$ and isentrope $\alpha$ profiles of the radiation preheated pellet at the time when the shock reaches the inner surface of the shell, demonstrating that the ablator can be put on a high isentrope, while the DT fuel is on a low isentrope. The laser pulse was shaped to produce high gain target performance. tungsten is in the range of $0.05-0.1 \mathrm{mg} / \mathrm{cm}^{2}$. Its radiation lasts approximately $5 \mathrm{~ns}$. Several ablator mixtures have been considered of the form $\mathrm{CH}(\mathrm{DT})_{\mathrm{n}}$. The optimized opacity is in the vicinity of $n \equiv 16$, so that most but not all of the ablator is heated directly by the soft x-rays from the tungsten.

Figure 14 plots the density $\rho$ and isentrope $\alpha$ versus radius at the time when the shock first breaks through the pellet shell. Note that the ablator material, $\mathrm{CH}(\mathrm{DT})_{16}$, has the high isentrope for good RT stabilization, while the DT fuel is on a very low isentrope. There is a small density jump between the ablator and the fuel, due to the radiation heating of the ablator. FAST2D calculations indicate that the laser imprinting is a factor of two less than the imprinting on the same target without a tungsten coating. Evaluation and optimization of this target design concept is ongoing at NRL to determine if a spatially varying isentrope $\alpha$ can provide sufficient RT stabilization while simultaneously maintaining the DT fuel on a very low-isentrope.

\section{Target with overcoats of foam and high- $Z$ shell.}

There is a fourth target concept that has not yet been fully developed. There has been a proposal to add a low-density "foam buffer" and a high- $Z$ outer coat to the outside of the ablator of an unspecified pellet. Prior to the usual foot of the laser pulse, there would be a separate laser pulse that heats the high- $Z$ material, producing $x$-rays that propagate supersonically through the low density foam and generate a long scale length corona on the ablator. The goal here is to reduce the laser imprinting. The use of supersonic radiation for this purpose was first proposed by $M$. Desselberger et al. in Ref. 25. More general suggestions to reduce the imprint with $x$-ray preheat were discussed earlier in Refs. 22 and ${ }^{60}$. 
Numerical simulations using FAST2D and ORCHID predict that an $x$-ray prepulse reduces but does not totally eliminate the laser imprinting. Proponents of the foam overcoat are now attempting to incorporate this concept into a selfconsistent ignition target design. (This design is fundamentally different from the one in Section $C$ that uses a high- $Z$ overcoat to preheat a DT-filled $\mathrm{CH}$ ablator to reduce the RT instability.)

\section{LASER-PLASMA INSTABILITIES}

Laser-plasma interactions can: (1) produce suprathermal electrons that preheat the target fuel and prevent high compression; (2) scatter the laser light away from the pellet, and reduce the coupling efficiency; (3) filament the laser light, thereby increasing the peak laser intensity and enhancing the other instabilities; (4) create denser structures in the underdense plasma that decouple the laser energy from the target.

We have completed an initial evaluation of the three major decay instabilities for the all-DT and all-CH foam ablator designs described in the previous Section. We utilized the predicted density, temperature, and velocity profiles for these pellets at various times during the implosion, and evaluated how far the designs were above or below the effective thresholds using models that assumed a coherent laser beam.

Currently three important elements are missing from the theoretical models. First, the total laser intensity includes all of the overlapped laser beams that are incident from different directions. For some instabilities we would expect these different laser beams to behave independently, which would then lower the effective laser intensity for driving laser-plasma instabilities. Second, the theories assume that the laser has a constant intensity. However these beams have both temporal and spatial fluctuations. For ISI beams the speckle satisfies Gaussian statistics ${ }^{61}$ (this is also true for broad-band SSD, except for the lower spatial frequencies). Under these conditions, the probability of observing a given intensity ${ }^{62}$ is $\exp (-$ $\left.I / I_{0}\right)$, where $I_{0}$ is the average intensity. For incoherent light, $4 \%$ of the laser energy is contained in intensities that are more than five times the average intensity. If this $4 \%$ of the laser energy is converted efficiently into fast electrons by, for instance, the two-plasmon decay mode, then the fuel preheat could be excessive. Third, decay instabilities can be driven out of resonance when the laser's frequency moves outside of the instability resonarice width; this resonance width is approximately the growth rate $\gamma$. For some of the decay instabilities, the growth rate is reduced ${ }^{63}$ from $\gamma$ to $\gamma^{2} / \Delta \omega$, when $\Delta \omega>\gamma$. (This is not true for all possible decay modes $^{64}$.) These three physics effects are in need of further theoretical and experimental research. The instability analyses in the following subsections should be viewed accordingly.

\section{A. Filamentation}

A laser beam can filament in a plasma, with an enhanced index of refraction induced by ponderomotive forces or thermal heating. This filamentation instability can be seeded at a finite amplitude by speckle in the laser beams. The growth of these speckles can then raise the peak laser intensities and enhance the various laserplasma instabilities. However with beam smoothing these laser speckles are constantly changing the hot spots, typically on a time scale of less than one picosecond, before they can undergo significant self-focusing. Because of this rapid time variation, along with a plasma corona size for direct drive targets of only a millimeter, only modest levels of time-dependence are sufficient to nearly quench the filamentation instability. 
Filamentation should not be important for direct drive targets, using either SSD or ISI. There are theoretical ${ }^{65}$ and numerous experimental studies ${ }^{66}$, $67,68,69,70,71,72,73$ that support this conclusion.

\section{B. Stimulated Raman Backscatter}

Stimulated Raman Scatter (SRS) is the decay of an electromagnetic wave into a plasma wave and a backscattered electromagnetic wave. Assuming a linear density profile, Raman backscattering is a convective instability except near the quarter critical density where it merges with the twoplasmon decay mode to form a high frequency, mixed polarization, absolute instability ${ }^{4}$. For densities below $n_{c} / 4$, the SRS backscattering threshold for significant convective gain ${ }^{75,76}$ is given by

$$
I^{S R S} \geq \frac{40}{L_{N} \lambda_{0}}
$$

where $I$ is in units of $10^{14} \mathrm{~W} / \mathrm{cm}^{2}, L_{\mathfrak{n}}$ is the density scale length in units of $100 \mu \mathrm{m}$, and $\lambda_{0}$ is the laser wavelength in units of $\mu \mathrm{m}$.

Using this formula, the two direct-drive targets we have considered are below threshold for convective SRS growth through the entire under dense plasma, at all times during the implosion, because of the relatively large density gradients and the relatively modest peak laser power with direct-drive targets. This does not exclude the possibility of SRS occurring during the timedependent statistical variations of the speckle associated with beam smoothing, or from local maxima or minima (Ref. 60 ) induced by other instabilities such as the RT instability or the twoplasmon decay mode ${ }^{\pi}$. (Recent unpublished OMEGA pellet experiments without beam smoothing have shown negligible SRS when several beams are incident from different directions.)

\section{Stimulated Brillouin Scatter}

Stimulated Brillouin Scatter (SBS) is the decay of an electromagnetic wave into an ion acoustic wave and a backscattered electromagnetic wave. In practical units the SBS threshold for significant convective growth is 74,75

$$
I^{S B S} \geq 1.7 \frac{T_{e}}{\left(n / n_{c}\right) L_{u} \lambda_{0}}
$$

where $L_{u}$ is the velocity scale length in units of 100 $\mu \mathrm{m}$, and $T_{e}$ is the electron temperature in $\mathrm{keV}$.

This formula predicts that the two targets considered would be above threshold, if the laser intensity is the sum of all of the beams. The maximum level of instability would occur as the laser pulse first rises from the foot to the main pulse; this condition has a low temperature and a high laser intensity. For the all-DT target with $1 / 3$ $\mu \mathrm{m}$ light, $I / I_{S B S}$ reached a value of 6 at a density of $0.2 n_{c}$. For the $\mathrm{CH}$-foam ablator with $1 / 4 \mu \mathrm{m}$ light, $I / I_{S B S}$ reached a values of $\sim 1$ at the transition to the main pulse.

The above analysis used the velocity gradients predicted for direct drive targets. Although the gradients are gentle enough so that the instability threshold can be exceeded, the gradients are also large enough to dominate the physics. There is the possibility that the temporal variations of the laser speckle pattern may drive acoustic waves that stimulate the SBS instability.

Each spot on the surface of the pellet is illuminated by many laser beams, which raises the question of power transfer between beams. This effect may be important for other types of laser fusion targets ${ }^{78}$ that require beam phasing and beam overlap at very high laser intensities. However for direct-drive this power transfer should not be important, because of the excellent power balance between beams, and because of the temporal and spatial incoherence between the beams. 


\section{PREPRINT}

The above analysis does not include any plasma fluctuations that would arise from the temporal and spatial incoherence in the laser beams, or long wavelength fluctuations due to other instabilities such as RT and the two plasmon decay, which may stabilize SBS (whereas they can destabilize SRS as noted above).

One can not yet conclude that there will or will not be significant levels of SBS instability in these pellets. This will be a subject for further experimental and theoretical research.

\section{Two-Plasmon Decay}

The two-plasmon instability is the decay from an electromagnetic wave into two electron plasma waves, and occurs near quarter critical density ${ }^{79}$. In practical units, the threshold condition is

$$
I^{2 \omega_{p}} \geq 0.54 \frac{T_{e}}{L_{N} \lambda_{0}}
$$

With the above formula, this instability is potentially very dangerous for both pellet designs. However the peak growth rate of this instability for short wavelength lasers has a long transverse wavelength, and it may be quenched by the spatial incoherence in the laser beams. Given that the threshold is so low, many modes will be driven unstable and the most dangerous modes may be those that are not most resonant, but which suffer large Landau damping and extend to densities well below $n_{c} / 4$. Such modes will no longer preserve the geometry of the most unstable, highly resonant modes, but can slowly grow and convectively saturate at high levels long after the more resonant modes have saturated nonlinearly. However these non resonant modes with low growth rate may be more easily stabilized by the broad bandwidth of lasers with ISI or SSD.

The above analysis is supported by recent highintensity OMEGA pellet experiments that used unsmoothed laser beams. There was no evidence for the resonant two-plasmon mode when multiple beams were overlapped, but there was evidence of the non resonant modes.

This instability has the potential of generating harmful energetic electrons through Landau damping and wave breaking of plasma waves. Neither the details of this instability nor the electron generation process are well understood. Further studies are necessary, to include the effects of multiple laser beam overlap with broad bandwidth light, and the probabilistic variation in laser intensity.

\section{E. Radiating Plasma Structures}

If a target contains material other than DT, then radiation can have two effects. On the positive side, it can reduce the density gradients and reduce the RT growth rate. On the negative side, computational modeling with highly-resolved opacity data sometimes predicts the occurrence ${ }^{80}$ of Radiating Plasma Structures (RPS). These RPS are related to the radiative condensation instability ${ }^{81}$. They occur when the laser intensity is a function of time, and the function $L=4 \sigma \kappa_{p} \rho T^{4} / \rho^{2}$ goes through a maximum as a function of $T$, where $\sigma$ is the Stefan-Boltzmann constant, $\rho$ is the density, and $\kappa_{p}$ is the mean Planck opacity. Then plasmas at a lower temperature can radiate more than adjacent plasmas at a higher temperature. The lower temperature material forms a local higher density region that can then interfere with the laser propagation. For carbon, the main effect occurs when the electron temperature is in the vicinity of $65 \mathrm{eV}$, and the $\mathrm{K}$-shell of carbon is excited. This RPS can complicate the use of some otherwise desirable laser pulse shapes. The structures are sometimes seen in radiation-hydrodynamics codes, but have not yet been directly observed in experiments.

F. Fokker-Planck Modifications to Thermal Transport 
The computer models that are used to design targets assume that thermal transport satisfies a Spitzer-Härm diffusion equation. However it has been noted ${ }^{82,83,84,85,86,87}$ that even if the electron distribution function is a Maxwellian in the absorption region, the electrons in the tail can have a mean free path that reaches farther than the thermal diffusion front, producing preheat perhaps into the DT fuel. There has been a recent attempt to model these kinetic effects on ignition target design ${ }^{88}$, but improvements will be needed in the modeling. We therefore can not yet quantitatively evaluate the potentially deleterious effects of this fast electron preheat.

\section{LASERS FOR A REACTOR}

For a fusion reactor using direct-drive pellets, the two lasers currently under consideration are a $\mathrm{KrF}$ laser and a DPSSL. These lasers will have to satisfy severe requirements in efficiency, repetition rate, cost, durability, and laser beam quality. Neither system can now meet all of the requirements, but the advocates of the two lasers have proposed solutions that may make them viable. Because of the modular design of fusion lasers, consisting of many parallel beam lines, it should be possible to develop and evaluate both laser concepts with modest levels of funding.

As an example, a KrF driver could utilize 100 large amplifiers, each producing $34 \mathrm{~kJ}$ of laser energy. Each of these amplifiers in turn would be pumped by four identical electron beam systems, each producing an "equivalent" of $8.5 \mathrm{~kJ}$. This core module would be only slightly larger than the 5-6 kJ achieved with the Nike laser at NRL. The existing pulsed power technology would be replaced by efficient and reliable non-linear magnetic switches similar to the Repetitive High Energy Pulsed Power device ${ }^{89}$ (RHEPP), or the recently developed Semiconductor Opening
Switch $^{90}$ (SOS). The optical design of a $\mathrm{KrF}$ fusion laser could be similar to Nike, and it would use the same type of gas mixture of $\mathrm{Ar} / \mathrm{Kr} / \mathrm{F}_{2}$.

For the DPSSL, the Xe flashlamps used in lasers such as OMEGA and the NIF would be replaced by efficient and reliable semiconductor diode arrays". The Nd:glass lasing medium would be replaced by a rare-earth doped crystal with a longer lived upper state and an absorption band chosen to effectively use the diode arrays. Turbulent gas cooling has been proposed to cool the laser crystals. The laser architecture would use the same type of multi-pass extraction as the NIF.

\section{A. Efficiency}

The reactor study called Sombrero ${ }^{92}$ estimated the total efficiency of a $\mathrm{KrF}$ laser to be $7.5 \%$, plus $2 \%$ heat recovery from the laser gas. This requires a $75 \%$ efficient pulsed power system, compared to the existing $60 \%$ of RHEPP and $50 \%$ of SOS.

Reference 91 presents a design for a DPSSL with a total laser efficiency of $8.6 \%$. This study assumed a diode efficiency of $55 \%$, improved from the level of current diodes ${ }^{93}$ of $45-50 \%$. There is promising recent research ${ }^{26}$ that has raised the diode efficiencies to as high as $66 \%$.

\section{B. Repetition Rate}

The diode pump should easily run at a high repetition rate. In fact, a higher repetition rate increases the duty cycle of the diodes, and thus reduces their capital cost in comparison to the fusion power output. However the beam quality of a DPSSL under appropriate loading must still be proven. This will be evaluated as part of the new Mercury project at LLNL (Lawrence Livermore National Laboratory) that utilizes face pumping and face cooling with turbulent helium-gas flow ${ }^{95}$.

For $\mathrm{KrF}$, the RHEPP runs at $120 \mathrm{~Hz}$, far above the $5-7 \mathrm{~Hz}$ needed for a reactor. It uses all solid state 


\section{PREPRINT}

components and should also have an extremely long lifetime $\left(>10^{9}\right.$ shots). The SOS has a repetition rate of $150 \mathrm{~Hz}$. The lifetime is reported to be very long. The EMRLD electron-beam pumped excimer laser has already demonstrated ${ }^{96}$ amplification of a laser beam at only 1.3 times diffraction limited at $100 \mathrm{~Hz}$.

\section{Cost}

The current cost of $\mathrm{KrF}$ power supplies would be dominated by the high price of Metglass that is used in the saturable inductors. At the current cost of $\$ 100 / \mathrm{kg}$, the cost of a $\mathrm{KrF}$ laser would be too high by a factor of about 40 . However Metglass is slowly being adopted by the power industry, and costs are predicted to drop significantly. (The same assumption is used in the design of heavy ion accelerators for a fusion reactor.)

The current cost of laser diodes is in excess of 10 $\$ / W$, too high by a factor of 100 . The cost is currently high because production rates are low. Costs should dramatically reduce as demand increases and processing steps become more automated. Newer diode arrays are being developed that are more compatible with automation. Scaling laws predict costs of 0.10 $\$$ /peak-Watt with production levels appropriate to one reactor per year. If costs drop well below 0.10 $\$ /$ peak-Watt, then one could use more diodes per laser disk, and pump the laser for a shorter period of time. There would then be less spontaneous decay from the excited state, and a resulting higher laser efficiency. Efficiencies as high as $15 \%$ are believed possible if the cost of the diodes becomes negligible compared to the other reactor components.

\section{Durability}

A very attractive feature of the DPSSL driver is its durability. Major components are expected to last $>10^{9}$ shots, except for routine repolishing and replacement of optical components.

$\mathrm{KrF}$ lasers are now durable enough for laser fusion research, but not for a power plant. The two major durability problems with the Nike laser are the lifetime of the thin foils that separate the laser gas cell from the electron beam source, and the lifetime of the anti-reflection coating on the windows of the laser cell. However longer foil lifetimes were achieved on the EMRLD laser; their foils did not degrade ${ }^{96}$ even after $10^{5}$ shots. The anti reflection-coated laser windows operate in a rather hostile environment of fluorine, intense UV laser light, $x$-rays, and energetic electrons. The coatings on the fused silica windows of the large Nike amplifier degrade after a few hundred laser shots. We do not yet know the reason for this degradation. Solutions have been proposed, such as the use of fluorine resistant $\mathrm{CaF}_{2}$ windows, and it is the subject of low-level ongoing research.

\section{E. Laser Beam Uniformity}

Successful pellet implosions require optical beam smoothing in both the high transverse modes and the low transverse modes. A KrF laser with ISI provides the maximum level of beam smoothing in both regimes, because it can have a large bandwidth, up to $3 \mathrm{THz}$, and because the "beamlets" in ISI are all statistically independent. Nike has already demonstrated the high-mode beam uniformity that would be necessary for a fusion reactor, with $1 \%$ rms fluctuations in one beam in a $4 \mathrm{~ns}$ pulse at $1 \mathrm{THz}$. This extrapolates to $-0.15 \%$ rms with 39 overlapping beams. There has also been occasional operation at $3 \mathrm{THz}$.

The proposed solid state lasing medium, $\mathrm{Yb}^{3+}$ doped $\mathrm{Sr}_{5}\left(\mathrm{PO}_{4}\right)_{3} \mathrm{~F}$, (or $\mathrm{Yb}: \mathrm{S}-\mathrm{FAP}$ ), has a narrow line width, producing only $\sim 0.3 \mathrm{THz}$ at the third harmonic. This crystal was chosen to maximize the diode's pump duration while still producing 


\section{PREPRINT}

adequate gain ${ }^{97}$. There are other possible hosts ${ }^{98}$ for $\mathrm{Yb}$, including Ca substituted $\mathrm{Yb}: S-F A P$ that would have three times the bandwidth. However the Einstein relationship shows that a higher bandwidth implies a higher saturation fluence. Changing to this higher bandwidth host would increase the saturation fluence from $3.2 \mathrm{~J} / \mathrm{cm}^{2}$ to $6.0 \mathrm{~J} / \mathrm{cm}^{2}$. Since efficient extraction of the inversion requires a laser fluencethat is about three times the saturation fluence, this would require higher damage threshold optical components.

The beam uniformity of 2D-SSD has so far been limited by the bandwidth of the frequency converter, and the use of a single color cycle, which does not provide temporal smoothing of the lowest mode numbers. The frequency conversion bandwidth may be increased by using advanced two-tripler schemes ${ }^{14}$, and the low-mode distortion may be reduced by increasing the number of color cycles across the beam ${ }^{16}$. The potential of advanced two-tripler schemes and multiple color cycling is under investigation.

\section{PROSPECTS FOR FUSION}

Solutions have been developed to the problems in direct-drive of laser uniformity, hydrodynamic instability, and laser-plasma instabilities. There are now three specific target designs that may meet all of the physics constraints and achieve ignition. The target design codes have extensive physics packages, and they have been successfully compared with numerous laser-target experiments using a variety of sophisticated diagnostics. The remaining disagreements may be attributable to 3D effects that are not in the 2D codes, and that probably require that the $2 \mathrm{D}$ target designs contain a margin of safety for larger-than predicted RT saturation effects. Two of the target designs keep the DT fuel on a low enough isentrope so that gains above 100 may be possible. All three target designs may be applicable to the NIF laser that is now under construction.

Although the prospects for ignition with direct drive are now very promising, there are also some remaining uncertainties. The Rayleigh-Taylor instability needs to be further evaluated in planar geometry with the specific ablator materials that would be used in ignition targets. The RayleighTaylor instability should also be evaluated in spherical geometry, to evaluate higher laser intensities and lower-mode perturbations. Laserplasma instabilities need re-valuation with statistically smooth laser beams to examine the roles of laser beam overlap and laser bandwidth. $\mathrm{A}$ larger data base is needed to better evaluate the new non-LTE models of opacity and emissivity. The design of the three targets is still incomplete, with research especially needed into Fokker-Planck effects, 3D RT saturation, final deceleration and burn, and non-LTE modeling of emissivity. Development has begun on a rep-rate DPSSL, and a parallel program should be started to develop a rep-rate $\mathrm{KrF}$ laser.

The different capabilities of Nike and OMEGA complement each other. The Nike $\mathrm{KrF}$ laser has the most uniform laser beam, and was optimized for a long pulse duration and modest laser intensity to study laser imprinting and the Rayleigh-Taylor instability in planar geometry scaled from fusion targets. The OMEGA Nd:glass laser with $3 \omega$ frequency conversion is the first fusion laser with a sufficient number of laser beams to properly illuminate and symmetrically implode a spherical pellet, and it can more easily reach the higher laser intensities needed for the study of laser-plasma instabilities.

\section{ACKNOWLEDGMENTS}

For the radiation-preheated pellet design, Avraham Bar-Shalom and Marcel Klapisch 
identified several atoms that can produce radiation in the low opacity window in carbon, and recommended tungsten as an easily available material. The work at the Naval Research Laboratory was supported through an interagency agreement with the Department of Energy, and by the Office of Naval Research. The work at the University of Rochester was supported by the US Department of Energy Office of Inertial Confinement Fusion under Cooperative Agreement No. DE-FC03-92SF19460, the University of Rochester, and the New York State Energy Research and Development Authority. The work at Polymath Assoc. was supported by the US Departmient of Energy through a subcontract with the Naval Research Laboratory. The work at Lawrence Livermore National Laboratory was supported by the U.S. Department of Energy under Contract No. W-7405-ENG-48.

J. Nuckolls, L. Wood, A. Thiessen, and G. Zimmerman, Nature 239, 139 (1992)

Laboratory for Laser Energetics LLE Review 37, p. 6 (Oct-Dec 1988), available from NTIS, Springfield VA.

${ }^{3}$ A. J. Schmitt, Appl. Phys. Lett. 44, 399 (1984)

A. J. Schmitt \& J. H. Gardner, J. Appl. Phys. 60, 6 (1986); S.

Skupsky \& K. Lee, J. Appl. Phys. 54, 3671 (1983)

R. H. Lehmberg and S. P. Obenschain, Optics Comm. 46, 27 (1983)

R. H. Lehmberg and J. Goldhar, Fusion Technol. 11, 532 (1987) R. H. Lehmberg, A. J. Schmitt, and S. E. Bodner, J. Appl. Phys. 62, $2680(1987)$

T. Lehecka, R. H. Lehmberg, A. V. Denix, K. A. Gerber, S. P. Obenschain, C. J. Pawley, M. S. Pronko and C. A. Sullivan, Optics Comm. 117, 485 (1995)

D. Véron, A. Ayral, C. Gouédard, D. Husson, J. Lauriou, 0. Martin, B. Meyer, M. Rostaing and C. Sauteret, Optics Comm. 65, 42 (1988); D. Véron, G. Thiell and C. Gouédard, Optics Comm. 97, 259 (1993

S. P. Obenschain, S. E. Bodner, D. Colombant, K. Gerber, R. H. Lehmberg, E. A. McLean, A. N. Mostovych, M. S. Pronko, C. J. Pawley, A. J. Schmitt, J. D. Sethian, V. Serlin, J. A. Stamper, C. A. Sullivan, J. P. Dahlburg, J. H. Gardner, Y. Chan A. V. Deniz, J. Hardgrove, T. Lehecka, and M. Klapisch, Phys. Plasmas 3, 2098 (1996)

UB. M. Van Wonterghem, C. S. Barker, S. C. Burkhart, J. H. Campbell, J. R. Murray, L. Smith, and D. R. Speck, in 1995 Baltimore Conference on Lasers and Electro-Optics, vol. 15, (OSA Technical Digest Series, Washington, DC, 1995)

A. V. Deniz, T. Lehecka, R. H. Lehmberg, and S. P.

Obenschain, Optics Comm., in press

'S. Skupsky, R. W. Short, T. Kessler, R. S. Craxton, S. Letzring and J. M. Soures, J. Appl. Phys. 66, 3456 (1989)

Laboratory for Laser Energetics LLE Review 45, pp 1-12
(1990); available from NTIS DOE/DP40200-149

D. Eimerl, I. M. Auerbach, C. E. Barker, D. Milam, and P. W. Milonni, Optics Lett. 22, 1208 (1997)

5. E. Rothenberg, J. Opt. Soc Am, B14, 1664 (1997)

D. Veron, G. Thiell, and C. Gouedard, Optics Comm. 97, 259 (1993)

H. Nakano. K. Tsubakimoto, N. Miyanaga, M. Nakatsuka, T. Kanabe, H. Azechi, T. Jitsuno, and S. Nakai, J. Appl. Phys. 73, 2122 (1993)

O.K. Hoffer, L. R. Foreman, J. J. Sanchez, E. Mapoles, and J. D. Sheliak, Fusion Tech. 30, 529 (1996)

A. I. Nikitenko, S. M. Tolokonnikov, and R. Cook, Fusion

Tech. 31, 385 (1997)

${ }^{2}$ L. Foreman, Los Alamos National Laboratory, private communication

M. H. Emery, J. H. Gardner, R. H. Lehmberg, and S. P.

Obenschain, Phys. Fluids B3, 2640 (1991)

${ }^{2}$ R. J. Taylor, A. L. Velikovitch, J. P. Dahlburg, J. H. Gardner, Phys. Rev. Lett 79, 1861 (1997)

${ }^{2}$ A. L.Velikovich, J. P.Dahlburg, J. H.Gardner, and R. J.Taylor, "Saturation of perturbation growth in ablatively driven planar laser targets," submitted to Phys.Plasmas (Dec, 1997)

${ }^{2} \mathrm{M}$. Desselberger, M. W. Jones, J. Edwards, M. Dunne, and $O$. Willi, Phys. Rev. Lett. 74, 2961 (1995); M. Durune, M. Borghesi, A. Iwase, M. W. Jones, R. Taylor, O. Willi, R. Gibson, S. R. Goldman, J. Mack, and R. G. Watt, Phys. Rev. Lett. 75, 3858 (1995)

A. B. Bud'ko and M. A. Liberman, Phys. Fluids B4, 3499 (1992)

tS. Bodner, Phys. Rev. Lett. 33, 761 (1974)

${ }^{2} \mathrm{H}$. Takabe, K. Mima, L. Montierth, and R. L. Morse, Phys. Fluids 28, 3676 (1985)

${ }^{2}$ G. R. Baker, R. L. McCrory, C. P. Verdon, and S. A. Orszag, Jour. Fluid Mech. 178, 165 (1987)

${ }^{M}$ C. P. Verdon, R. L. McCrory, R. L. Morse, G. R. Baker, D. I.

Meiron, and S. A. Orszag, Phys. Fluids 25, 653 (1982)

${ }^{31}$ R. L. McCrory, L. Montierth, R. L. Morse and C. P. Verdon, in

"Laser Interaction and Related Phenomena", vol. 5, p. 713

(Plenum Pub, NY, 1981)

7. H. Gardner, S. E. Bodner, and J. P. Dahlburg, Phys. Fluids B 3, 1070, (1991)

C. P. Verdon, R. L. McCrory, R. L. Morse, G. R. Baker, D. I.

Meiron, and S. A. Orszag, Phys. Fluids 25, 1653 (1982)

${ }^{34}$ H. J. Kull and S. Anisimov, Phys. Fluids 29, 2067 (1986)

${ }^{3}$ H. J. Kull, Phys. Fluids B1, 170 (1989)

${ }^{3}$ R. Betti, V. N. Goncharov, R. L. McCrory, and C. P. Verdon, Phys. Plasmas 2, 3844 (1995)

V. N. Goncharov, R. Betti, R. L McCrory, P. Sorotokin, and C. P. Verdon, Phys. Plasmas 3, 1402 (1996)

${ }^{3}$ R. Betti, V. N. Goncharov, R. L. McCrory, P. Sorotokin, and C. P. Verdon, Phys. Plasmas 3, 2122 (1996)

V. V. N. Goncharov, R. Betti, R. L. McCrory, and C. P. Verdon, Phys. Plasmas 3, 4665 (1996)

A. R. Piriz, J. Sanz, and L. F. Ibanez, Phys. Plasmas 4, 1117 (1997)

S. Atzeni, Nucl. Fusion 36, 69 (1996)

9. Sanz, Phys. Rev. E 53, 4026 (1996)

R. Betti, V. N. Goncharov, R. L. McCrory, and C. P. Verdon,

"Growth rates of the ablative Rayleigh-Taylor instability in

inertial confinement fusion", submitted to Phys. Plasmas

S. W. Haan, Phys. Fluids B3, 2349 (1991)

D. Ofer, U. Alon, D. Shvarts, R. L. McCrory, and C. P. Verdon, Phys. Plasmas, 3, 3073 (1996)

J. Hecht, D. Ofer, U. Alon, D. Shvarts, S. A. Orszag, and R. L. McCrory, Phys. Plasmas 2, 2465 (1995)

D. Shvartz, U. Alon, D. Ofer, R. L. McCrory, and C. P.

Verdon, Phys. Plasmas 2, 2465 (1995)

D. Ofer, J. Hecht, D. Shvartz, Z. Zinamon, S. A. Orszag, and R. L. McCrory, AIP Conf Proc., American Institute of Physics, Woodbury, NY, 314, 26 (1994) 
For a discussion of the ORCHID computer model, see R. L.

McCrory and C.P. Verdon in Computer Applications in

Plasma Science and Engineering, pp. 291-325, edited by Adam

T. Drobot (Springer-Verlag, New York, 1991)

C. J. Pawley, K. Gerber, R. H. Lehmberg, E. A. McLean, A. N.

Mostovych, S. P. Obenschain, J. D. Sethian, V.Serlin, J. A.

Stamper, C. A. Sullivan, S. E. Bodner, D. Colombant, J. P.

Dahlburg, A. J. Schmitt, J. H. Gardner, C. Brown, J. F. Seely, T.

Lehecka, Y. Aglitskiy, A. V. Deniz, Y. Chan, N. Metzler, and M.

Klapisch, Phys. Plasmas 4, 1969 (1997)

${ }^{5}$ For a discussion of the FAST computer codes, and the recent Nike acceleration experments, see f. Gardner, Bull: Am Phys. Soc. 42, 1941 (1997)

sL. Spitzer and R. Härm, Phys. Rev. 89, 997 (1953)

7y. P. Dahlburg and J. H. Gardner, Phys. Rev. A 41, 5695 (1990)

J. P. Dahlburg, J. H. Gardner, D. D. Doolen, and S. W. Haan,

Phys. Fluids B 5, 571 (1993)

J. P. Dahlburg, D. E. Pyfe, J. H. Gardner, S. W. Haan, S. E.

Bodner, and G. D. Doolen, Phys. Plasmas 2, 2453 (1995)

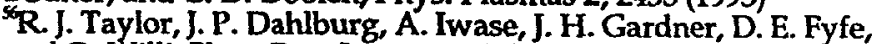

and O. Willi, Phys. Rev. Lett. 76, 1643 (1996)

Y. Aglitskiy, T. Lehecka, A. Deniz, J. Seely, C. Brown, U.

Feldman, C. Pawley, K. Gerber, S. Bodner, S. Obenschain, R

Lehmberg, E. McLean, M. Pronko, J. Sethian, J. Stamper, A.

Schmitt, C. Sullivan, G. Holland, and M. Laming, Rev. Sci. Instrum. 68, 806 (1997)

${ }^{588}$ C. Verdon, Bull. Am Phys. Soc. 38, 2010, (1993)

9M. Klapisch, Bull. Am Phys. Soc. 42, 2060, (1997)

S. Eliezer, J. J. Honrubia, and J. J. Velarde, Phys. Lett. A166 249 (1992)

J. J. W. Goodman, "Statistics of laser speckle patterns" in Laser Speckle and Related Phenomena, Ch. 2, ed. J. C. Dainty (Springer, Berlin, 1984)

S. P. Obenschain, C. J. Pawley, A. N. Mostovych, J. A.

Stamper, J. H. Gardner, A.J. Schmitt, and S. E. Bodner, Phys.

Rev. Lett. 62, 728 (1989); H. A. Rose \& D. F. Dubois, Phys. Fluids B5, 590 (1993)

I. J. Thomson, and J. I. Karush, Phys. Fluids 17, 1608 (1974)

T. N. Guzdar, C. S. Liu, and R. H. Lehmberg, Phys. Fluids B 3 , 2882 (1991)

${ }^{6}$ A. J. Schmitt, Phys Fluids 31, 3079 (1988); Phys. Fluids B3, 186 (1991)

W. Seka, R. E. Bahr, R. W. Short, A. Simon, R. S. Craxton, D. S. Montgomery, and A. E. Rubenchik, Phys. Fluids B4, 2232 (1992)

S. P. Obenschain, J. Grun, M. Herbst, K. Kearney, C. Manka, E. McLean, A. Mostovych, J. Stamper, R. Whitlock, S. Bodner, J. Gardner, and R. Lehmberg, Phys. Rev. Lett. 56, 2807 (1986)

A. Mostovych, S. Obenschain, J. Gardner, J. Grun, K. Kearney, A. Mostovych, E. McLean, and C. Pawley, Phys. Rev. Lett. 59, 1193 (1987)

S. P. Obenschain, C. Pawley, A. Mostovych, J. Stamper, J.

Gardner, R. Lehmberg and S. Bodner, Phys. Rev. Lett. 62, 768

(1989); T. Lehecka, R. H. Lehmberg, A. V. Deniz, K. A. Gerber, S. P. Obenschain, C. J. Pawley, M.S. Pronko, and C. A. Sullivan, Optics Comm. 117, 485 (1995)

r. Peyser, A. Mostovych, S. Obenschain, and K. Keamey, Phys. Fluids B3, 1479 (1991)

"C. LaBaune, S. Baton, T. Jalinaud, H. A. Baldis, D. Pesme, Phys. Fluids B4, 2224 (1992)

C. Rousseaux, B. Meyer and G. Thiell, Phys. Plasmas 2, 2075 (1995)

D. S. Montgomery, J. D. Moody, H. A. Baldis, B. B. Afeyan, R. L. Berger, K.G. Esterbrook, B. F. Lasinski and E. A. Williams, Phys. Plasmas 3, 1728 (1996)

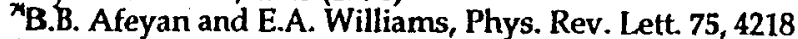

(1995), and Phys. Plasmas 4, 3845 (1997)

M.N. Rosenbluth, Phys. Rev. Lett. 29, 565 (1972)

"C.S. Liu, in "Advances in Plasma Physics", vol. 16, pg. 121,

(A. Simon and W. Thompson eds. Interscience; NY, 1976)
7B.B. Afeyan and E.A. Williams, Phys. Plasmas, 4, 3788 (1997), and 4, 3803 (1997)

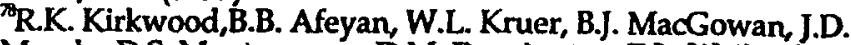
Moody, D.S. Montgomery, D.M. Pennington, T.L. Weiland, and S.C. Wilks, Phys. Rev. Lett. 76, 2065 (1996)

B.B. Afeyan and E.A. Williams, Phys. Plasmas 4, 3827 (1997) and 4, 3845 (1997)

J. P.Dahlburg, M. Klapisch, J. H. Gardner, C. R. DeVore, A. J. Schmitt, and A. Bar-Shalom, J. Quant. Spectrosc. Radiat. Transfer 54113 (1995)

E. N. Parker, Astrophys.J. 117, 431 (1953); G. Field, Astrophys 1. 142, 531 (1965)

A. R. Bell, R. G. Evans, and D. J. Nicholas, Phys. Rev. Lett. 46, 243 (1981)

\%. P. Matte, and J. Virmont, Phys. Rev. Lett. 49, 1936 (1982)

3. R. Albritton, Phys. Rev. Lett. 50, 2078 (1983)

T. F. Luciani, P. Mora, and J. Virmont, Phys. Rev. Lett. 51, 1664 (1983)

H. T. Kho, D. J. Bond, and M. G. Haines, Phys. Rev. A 28, 156 (1983)

A. R. Bell, Phys. Fluids 28, 2007 (1985)

M. Honda, A. Nishiguchi, H. Takabe, H. Azechi, and K

Mima, Phys. Plasmas 3, 3420 (1996)

\%. X. Schneider, Sandia Laboratories, private communication,

Yu. A. Kotov, G.A. Mesyats, S.N. Rukin, and A. L. Filatov, Digest of Technical Papers, Ninth IEEE Pulsed Power Conf., Albuquerque, pg. 134 (IEEE, NY, 1993)

"C.D. Orth, S.A. Payne, and W.F. Krupke, Nuclear Fusion 36, 75 (1996)

9. V. Sviatoslavsky, M. E. Sawan, R. R. Peterson, G. L. Kulcinski, J. J. MacFarlane, L. J. Wittenberg, H. Y. Khater, E. A. Mogahed, and S. C. Rugledge, Fusion Tech. 21, 1470 (1992)

${ }^{2}$ C. D. Marshall, L. K. Smith, R. J. Beach, M. A. Emanuel, K. I. Schaffers, J. Skidmore, S. A. Payne, and B. H. T. Chaî, IEEE J. Quantum Elect. 32, 650 (1996)

D. Botez, L. I. Mawst, A. Bhattacharya, J. Li, T. F. Kuech, V. P. Iakovlev, G. I. Suruceanu, A. Caliman, and A. V. Syrbu, " $66 \%$

CW wallplug efficiency from $\mathrm{Al}$-free $0.98 \mu \mathrm{m}$-emitting diode lasers", to be published in Electronics Letters

gf. L. Emmett, W. F. Krupke, and J. B. Trenholme, Soviet J. Quantum Electronics 13, 1 (1983)

\%. Moran, Textron Defense Systems, Everett, MA, private communication

"L. D. DeLoach, S. A. Payne, L. L. Chase, L. K. Sinith, W. L.

Kway, and W. F. Krupke, IEEE J. Quantum Electronios 29, 1179 (1993)

S. A. Payne, L. D. DeLoach, L. K. Smith, W. L. Kway, J. B.

Tassano, W. F. Krupke, B. H. T. Chai, and B. Loutts, J. Applied Phys. 76, 497 (1994) 